\title{
Integration of Constructed Floodplain Ponds into Nature-Like Fish Passes Supports Fish Diversity in a Heavily Modified Water Body
}

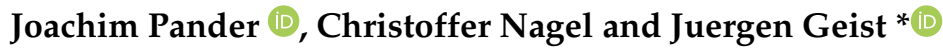

check for updates

Citation: Pander, J.; Nagel, C.; Geist, J. Integration of Constructed Floodplain Ponds into Nature-Like Fish Passes Supports Fish Diversity in a Heavily Modified Water Body. Water 2021, 13, 1018. https:// doi.org/10.3390/w13081018

Academic Editor: José Maria Santos

Received: 22 February 2021

Accepted: 4 April 2021

Published: 8 April 2021

Publisher's Note: MDPI stays neutral with regard to jurisdictional claims in published maps and institutional affiliations.

Copyright: (c) 2021 by the authors. Licensee MDPI, Basel, Switzerland. This article is an open access article distributed under the terms and conditions of the Creative Commons Attribution (CC BY) license (https:// creativecommons.org/licenses/by/ $4.0 /)$.
Aquatic Systems Biology Unit, Department of Life Science Systems, TUM School of Life Sciences, Technical University of Munich, Mühlenweg 22, D-85350 Freising, Germany; joachim.pander@tum.de (J.P.); christoffer.nagel@tum.de (C.N.)

* Correspondence: geist@wzw.tum.de; Tel.: +49-8161-713767; Fax: +49-8161-713477

\begin{abstract}
Fish passes facilitate fish movement in fragmented river systems, yet they can also provide important habitat functions. This study investigated the fish community composition of different constructed habitat types (fluvial habitats, floodplain ponds) within fish passes in relation to habitat characteristics in order to deduce recommendations for fish-friendly designs of such structures. Fish community structures within passes differed significantly from those in the main river, comprising a high number of rheophilic species in fluvial habitats (Thymallus thymallus, Hucho hucho, Salmo trutta, Cottus gobio, Chondrostoma nasus, and Barbus barbus), and of stagnophilic species in floodplain ponds (Rhodeus amarus, Scardinius erythrophthalmus, Misgurnus bipartitus, and Tinca tinca). During summer, floodplain ponds also provided important juvenile habitats for the target species C. nasus and B. barbus. Differences between the two habitat types in fish abundance were mostly explained by differences in macrophyte coverage, gravel, boulders, temperature, and current speed. The findings of this study stress the important habitat functions of fish passes. They also suggest that integration of diverse habitat structures, especially of currently hardly considered constructed floodplain ponds into fish passes, can greatly enhance their fish communities and contribute to the restoration of several declining target species of conservation.
\end{abstract}

Keywords: river restoration; bypass channel; fish conservation; aquatic habitat; floodplain ponds; hydropower; fish stock management; fish migration

\section{Introduction}

Habitat degradation and fragmentation of river systems caused by dams, weirs, and other barriers is considered a major challenge in restoring European fish populations [1]. For instance, impoundments that restrict river dynamic processes, such as sediment transport or other matter fluxes, have been identified as one of the major factors related to the decline of riverine fish species in the headwater areas of Elbe, Main, and Danube rivers $[2,3]$. Besides restoring habitat quality of rivers by improving structural richness, sediment transport and deadwood dynamics, the restoration of fish passage in fragmented rivers is high on the agenda [4-6]. Different techniques to restore fish migration, such as technical fish passes (e.g., vertical slot-pass; [7,8]) or solutions with nature-like construction schemes [9-11], have been applied in practice. These artificial structures have widely proven their valuable contributions to restore fish migration [12,13]. However, the restoration of fish communities in degraded rivers seems to be highly challenging and not only dependent on restoring connectivity, due to the manifold other restrictions in heavily modified water bodies (HMWB; [14]). In these waters, the natural river dynamic processes are strongly impaired [15-17], limiting the chances of restoring complete life cycles of specialized riverine fish species, such as Thymallus thymallus L., Hucho hucho L., Salmo trutta L., Chondrostoma nasus L., and Barbus barbus L. [18,19]. It has been proposed 
to restore fish habitats in highly degraded rivers by designing separate, parallel flowing river courses or channels outside the degraded systems using a portion of their natural discharge $[19,20]$ as it is required for nature-like fish passes that initially were built to restore fish migration (reviewed in [21]). This initiated a discussion between ecologists, river managers and users, to which extend fish passes can provide important fish habitats potentially compensating the existing habitat loss in the main river. First studies on the contribution of nature-like fish passes to overall biodiversity of fishes indicated that these structures can be highly valuable refuges for rheophilic fishes [13,19,22], e.g., providing key habitats for reproduction [11,12].

Many countries developed extensive guidelines how fish passes have to be built (e.g., Germany; [23]), often primarily focussing on their role for fish passage. Nature-like solutions are generally built to mimic small streams with discharges between $0.3 \mathrm{~m}^{3} / \mathrm{s}$ and $30 \mathrm{~m}^{3} / \mathrm{s}$ comprising runs, riffles and pools [23,24], with a special focus on providing a widely functional migration corridor for the target species of conservation throughout the year [23]. Besides the fluvial habitats, nature-like solutions increasingly consider the lateral connectivity to stagnant waters in the floodplain. These additional aquatic habitats can comprise large structures, such as backwaters that evolved from ancient cut-off meanders or small floodplain ponds [25-28]. If such structures are absent, they can also be newly constructed.

In this study, we investigated the value of fluvial habitats and newly created and connected small floodplain ponds (further referred to as ponds) as habitat for the fish community in two nature-like fish passes in relation to their characteristics. In particular, we hypothesized that (i) fish community composition in the nature-like fish passes reflects the fish community composition of the main stem, and (ii) nature-like fish passes integrating stagnant waters provide habitat for both rheophilic and stagnophilic fish species, thus contributing to greater fish diversity. Furthermore, we hypothesized that (iii) fish pass systems can provide important juvenile habitats for rheophilic and stagnophilic species, as well.

\section{Materials and Methods}

\subsection{Study Area}

The study was carried out at two nature-like fish passes at the hydropower plant Gars ( $\left.48^{\circ} 09^{\prime} 13.92^{\prime \prime} \mathrm{N}, 12^{\circ} 18^{\prime} 40.80^{\prime \prime} \mathrm{E}\right)$ and Stammham (48 $\left.14^{\prime} 50.67^{\prime \prime} \mathrm{N}, 12^{\circ} 51^{\prime} 57.03^{\prime \prime} \mathrm{E}\right)$, which are located at the heavily modified middle reach of the Bavarian Inn in Germany (Figure 1). The River Inn is a snow-melt dependent alpine river with relatively cold water temperatures $\left(2.0-17^{\circ} \mathrm{C}\right)$ and peak discharge in summer (mean annual discharge (MQ) in Summer $490 \mathrm{~m}^{3} / \mathrm{s}$ ) with turbid water (up to 200 nephelometric turbidity units (NTU)), and a clear water phase (often below 25 NTU) at low discharge (MQ $222 \mathrm{~m}^{3} / \mathrm{s}$ ) in winter. The River Inn is a highly altered alpine river due to flood protection and hydropower generation with many dams and 20 power plants. The loss of the former rich habitat mosaic comprising lentic floodplain habitats, as well as several river courses (Figure 2), impaired the recruitment of riverine fish species and severely reduced the amount of aquatic habitats of a water depth less than $1 \mathrm{~m}$. The interruption of the river continuum due to the implemented power plants additionally impaired the connectivity of life stage specific habitats, with strong effects on migratory fish populations, particularly the target species considered in this study. The degradation of the River Inn created a strong need to restore fish migration and aquatic habitats, as well, which was partly realized by implementing nature-like fish passes. 

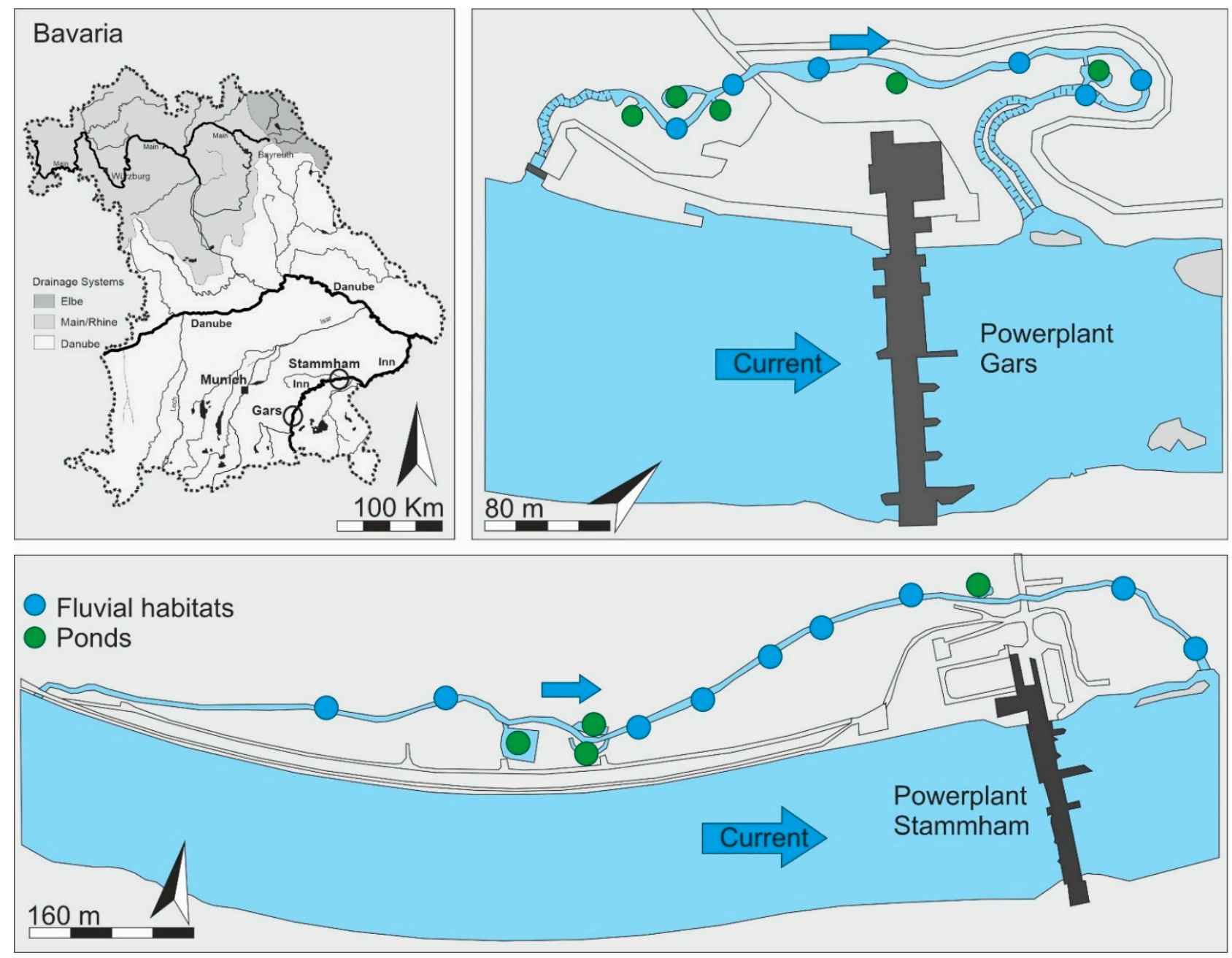

Figure 1. Map of Bavaria with the major rivers and drainage areas. Drainage system of the River Danube into which the River Inn drains is highlighted in light grey, with the location of the two fish passes Gars and Stammham, as well as the sampled fluvial habitats (blue) and ponds (green).
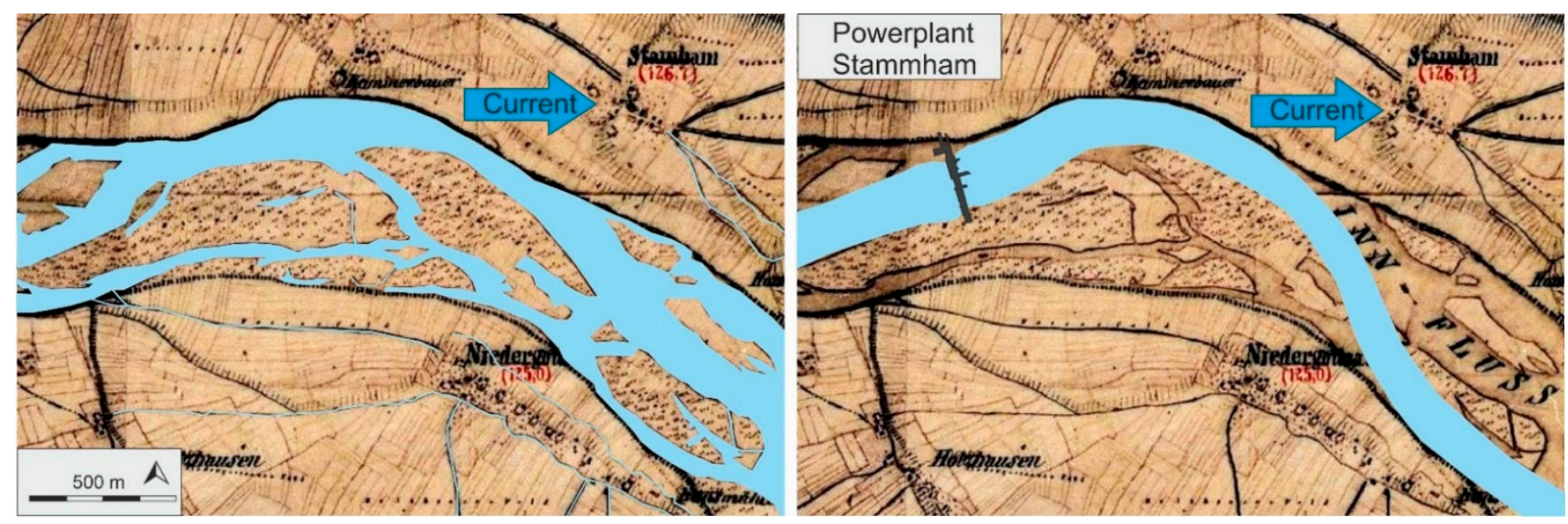

Figure 2. Illustration of the historic habitat mosaic of the River Inn comprising lentic as well as lotic habitats (left panel) compared the pre-restoration conditions after the implementation of the power plant Stammham in 1955 (right panel). The map was created based on the Bavarian "Positionsblätter", which dates back to the 1860s (https://geoportal.bayern.de/ bayernatlas $/$ ?lang=de\&topic=ba\&bgLayer=historisch\&catlogNodes=11,122\&E=786779.49\&N=5350979.79\&zoom=11; last accessed on 24 March 2021). For post-restoration conditions, please see Figure 1. 
The potential fish ecological region of the River Inn is the hyporhithral with historically widespread rheophilic specialists, such as T. thymallus, H. hucho, S. trutta, Cottus gobio L., C. nasus, and B. barbus [29], representing the largest part of the former natural fish community. Today, the rheophilic specialists are still present; however, their population density is rather low, and some of the more prominent species, such as T. thymallus and H. hucho, continue declining, although they are protected and stocked [30]. The fish community in the River Inn is now dominated by ubiquitous species, such as Alburnus alburnus L., Rutilus rutilus L., and Squalius cephalus L., as well as the stocked non-native Oncorhynchus mykiss Walbaum [30].

\subsection{Characterization of the Fish Passes}

Both fish passes (FP) were constructed with a nature-like construction scheme and finalized in 2015 (Gars) and 2016 (Stammham). With a length of $1500 \mathrm{~m}$, the FP Stammham is more than double the length of Gars $(670 \mathrm{~m})$; however, its discharge and width are much smaller (Tables 1 and 2).

Table 1. Construction details of the two assessed fish passes Gars and Stammham.

\begin{tabular}{ccccccc}
\hline Fish Pass & Year Built & Gradient [\%o] & Length [m] & $\begin{array}{c}\text { Height } \\
\text { Difference [m] }\end{array}$ & $\begin{array}{c}\text { Discharge } \\
{\left[\mathbf{L ~ s} \mathbf{~ s}^{-1}\right]}\end{array}$ & $\begin{array}{c}\text { Flow-Course } \\
\text { [m] }\end{array}$ \\
\hline Gars & 2015 & 10 & 670 & 7.5 & 900 & 670 \\
Stammham & 2016 & 5.5 & 1500 & 8.5 & 350 & 1500 \\
\hline
\end{tabular}

Table 2. Construction details of the fluvial habitats in Gars and Stammham. Values represent the mean and the range. CSB $=$ current speed above bottom, CSS = current speed below surface, MA = submerged macrophytes in $\%$ coverage, $\mathrm{DW}=$ deadwood in \% coverage, Veg = bank vegetation in \% coverage. $\mathrm{B}=$ boulder in $\%$ coverage, $\mathrm{G}=$ gravel in $\%$ coverage, $\mathrm{S}=$ sand in \% coverage, $\mathrm{F}=$ fines smaller $1 \mathrm{~mm} \%$ coverage.

\begin{tabular}{|c|c|c|c|c|c|c|c|c|c|c|c|c|}
\hline $\begin{array}{c}\text { Fluvial } \\
\text { habitats }\end{array}$ & $\begin{array}{c}\text { Width } \\
\text { [m] }\end{array}$ & $\begin{array}{c}\text { Depth } \\
{[\mathrm{m}]}\end{array}$ & $\begin{array}{c}\mathrm{CSB} \\
{\left[\mathrm{ms}^{-1}\right]}\end{array}$ & $\begin{array}{c}\mathrm{CSS} \\
{\left[\mathrm{ms}^{-1}\right]}\end{array}$ & $\begin{array}{l}\text { Area } \\
{\left[\mathrm{m}^{2}\right]}\end{array}$ & $\begin{array}{l}\text { MA } \\
{[\%]}\end{array}$ & $\begin{array}{l}\text { DW } \\
{[\%]}\end{array}$ & $\begin{array}{l}\text { Veg } \\
{[\%]}\end{array}$ & B [\%] & G [\%] & $\mathrm{S}[\%]$ & F [\%] \\
\hline \multirow{2}{*}{ Gars } & 4.83 & 0.43 & 0.43 & 0.61 & 146 & 2 & 5 & 7 & 34 & 67 & 23 & 11 \\
\hline & $\begin{array}{c}3.10-7.00 \\
2.89\end{array}$ & $\begin{array}{c}0.10-1.12 \\
0.34\end{array}$ & $\begin{array}{c}0.00-1.44 \\
0.36\end{array}$ & $\begin{array}{c}0.00-2.07 \\
0.41\end{array}$ & $\begin{array}{c}120-177 \\
89\end{array}$ & $\begin{array}{c}0-5 \\
3\end{array}$ & $\begin{array}{c}0-10 \\
5\end{array}$ & $\begin{array}{c}0-25 \\
46\end{array}$ & $\begin{array}{c}25-50 \\
4\end{array}$ & $\begin{array}{c}50-70 \\
60\end{array}$ & $\begin{array}{c}15-35 \\
26\end{array}$ & $\begin{array}{c}5-15 \\
14\end{array}$ \\
\hline Stammham & $1.83-4.32$ & $0.08-0.75$ & $0.00-1.09$ & $0.01-1.10$ & 75-107 & $0-10$ & $0-10$ & 0-90 & $0-25$ & $45-70$ & $20-35$ & $10-20$ \\
\hline
\end{tabular}

To create a high variability in aquatic habitats and to improve the fish diversity in the two fish passes, nine artificial ponds with different sizes and depth were built adjacent but connected to the flow course (Figures 1 and 3). In both FP, large stones and deadwood in form of dead rootstocks from forest clearance were placed for structural enrichment into the fluvial habitats, as well as into the small constructed ponds (Figure 3). The upstream connection of the FP was realized using an undershot sluice gate. At the upstream entrance of the FP Gars, a concrete structure $(8 \mathrm{~m} \times 4 \mathrm{~m})$ was built to remove frequently occurring high loads of silt and sand. In contrast to Stammham, where the discharge was fixed to $350 \mathrm{~L} / \mathrm{s}$ (comprising only water-level fluctuations in the $\mathrm{cm}$ range), the discharge in Gars (base flow of $900 \mathrm{~L} / \mathrm{s}$ ) can additionally be enhanced by applying environmental flows (e-flows; Reference [31]) of additional $900 \mathrm{~L} / \mathrm{s}$. This e-flow is mainly applied during the peak discharge phase of the River Inn in summer. Due to strongly fluctuating water levels in the tailwater of the power plant Gars, this FP was constructed with a second, parallel running fish passage structure of different height at the confluence to the River Inn. In Stammham, the downstream connection of the FP to the Inn was built like a natural tributary confluence (Table 1). The substrate introduced into the FP was gravel dominated with low percentage of sand and silt. In the ponds, accumulations of fine material built up to a thin layer of several $\mathrm{cm}(20-100 \mathrm{~mm})$ over the originally introduced gravel comprising a grain size range of 0-65 $\mathrm{mm}$. Depending on their depths, pond macrophyte coverage varied between $0-95 \%$. In fluvial habitats, macrophytes were widely absent. 

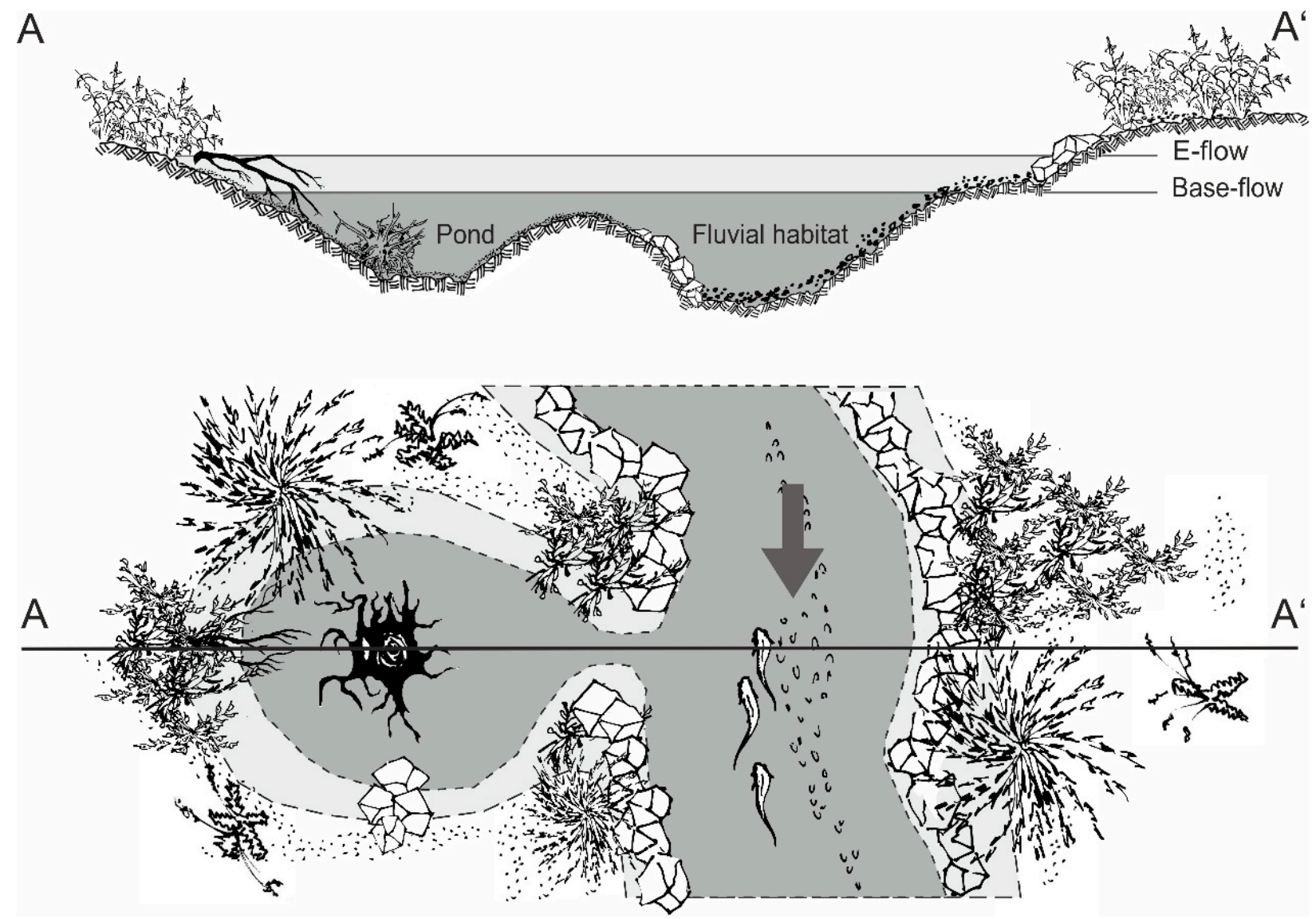

Figure 3. Schematic drawing (cross section and top-view) of the principle habitat types fluvial habitats and ponds, which were assessed in this study. Base-flow line indicates the water level at base-flow conditions $\left(900 \mathrm{~L} \mathrm{~s}^{-1}\right)$. E-flow line indicates water level during the application of environmental flows (additionally to the base-flow another $900 \mathrm{~L} \mathrm{~s}^{-1}$ discharge can be provided in the fish pass). Note that e-flow conditions can only be applied in the fish pass Gars.

\subsection{Fish Sampling}

Fish community composition in both FP and their adjacent ponds was assessed in spring (March, May), summer (June, August), and late fall (October, December) 2019. To assess a representative length of more than $20 \%$ of the FP (suggested in [32]), 6 fluvial habitats (FS) were sampled in Gars and 9 in Stammham. In both fish passes, all available floodplain ponds were fully sampled, altogether comprising 9 ponds of different size and depth (Table 3). Each of the 15 fluvial habitats comprised $30 \mathrm{~m}$ in length [32] and differed slightly in the sampled area according to the respective river width (Table 2). All fluvial habitats and ponds were sampled with a land-based electrofishing generator of $8 \mathrm{~kW}$ (EFKO FEG 8000, EFKO-Elektrofischfanggeräte GmbH, Leutkirch, Germany) during stable weather and discharge conditions. All fluvial habitats and ponds were consecutively sampled within a 6-h period (9 a.m.-3 p.m.), working from downstream to upstream direction. A single anode was used and stunned fish were collected with a dipnet and transferred to a plastic tank. During all sampling events, the same persons handled the anode and the dipnet. The total length of all specimens was measured to the nearest $\mathrm{cm}$. All individuals of lampreys, adults, as well as larvae, were determined to the family level Petromyzontidae. Fish and lampreys were immediately released after the length measurement at the location from which they had been collected. The same methodological approach was used across all sampling dates. 
Table 3. Construction details of the constructed floodplain ponds in Gars and Stammham. Values represent the mean and the range. $\mathrm{N}=$ number of ponds, $\mathrm{MA}=$ submerged macrophytes in $\%$ coverage, $\mathrm{DW}=$ deadwood in $\%$ coverage, Veg = bank vegetation in \% coverage. $\mathrm{B}=$ boulder in $\%$ coverage, $\mathrm{G}=$ gravel in $\%$ coverage, $\mathrm{S}=$ sand in $\%$ coverage, $\mathrm{F}=$ fines smaller $1 \mathrm{~mm} \%$ coverage. NA = not available.

\begin{tabular}{|c|c|c|c|c|c|c|c|c|c|c|c|c|c|c|}
\hline $\begin{array}{l}\text { Floodplain } \\
\text { Ponds }\end{array}$ & $\mathbf{N}$ & $\begin{array}{l}\text { Length } \\
\text { [m] }\end{array}$ & $\begin{array}{l}\text { Width } \\
\text { [m] }\end{array}$ & $\begin{array}{c}\text { Depth } \\
\text { Base- } \\
\text { Flow } \\
\text { [m] }\end{array}$ & $\begin{array}{c}\text { Depth } \\
\text { E-Flow } \\
{[\mathrm{m}]}\end{array}$ & $\begin{array}{l}\text { Area } \\
\text { Base- } \\
\text { Flow } \\
{\left[\mathbf{m}^{2}\right]}\end{array}$ & $\begin{array}{c}\text { Area } \\
\text { E-Flow } \\
{\left[\mathrm{m}^{2}\right]}\end{array}$ & $\begin{array}{l}\text { MA } \\
{[\%]}\end{array}$ & $\begin{array}{l}\text { DW } \\
{[\%]}\end{array}$ & $\begin{array}{l}\text { Veg } \\
{[\%]}\end{array}$ & B [\%] & G [\%] & $\mathrm{S}[\%]$ & $\mathbf{F}[\%]$ \\
\hline \multirow{2}{*}{ Gars } & \multirow{2}{*}{5} & 15.52 & 8.10 & 0.32 & 0.50 & 66 & 115 & 38 & 10 & 4 & 2 & 7 & 28 & 65 \\
\hline & & $\begin{array}{l}9.55- \\
25.89\end{array}$ & $\begin{array}{l}6.60- \\
9.52\end{array}$ & $\begin{array}{l}0.06- \\
0.93\end{array}$ & $\begin{array}{l}0.25- \\
1.08\end{array}$ & $44-130$ & 66-181 & 0-90 & $5-20$ & $0-20$ & $0-10$ & $0-15$ & $0-40$ & $50-90$ \\
\hline \multirow{2}{*}{ Stammham } & \multirow{2}{*}{4} & 26.00 & 11.70 & 0.51 & NA & 377 & NA & 58 & 8 & 4 & 3 & 13 & 26 & 61 \\
\hline & & $\begin{array}{l}11.47- \\
39.00\end{array}$ & $\begin{array}{l}2.89- \\
30.00\end{array}$ & $\begin{array}{l}0.17- \\
1.65\end{array}$ & NA & $\begin{array}{c}67- \\
1127\end{array}$ & NA & $10-95$ & 5-15 & $0-10$ & 0-10 & $0-40$ & $20-30$ & $30-75$ \\
\hline
\end{tabular}

For the fish community analyses of the River Inn, data from the governmental fish monitoring in context of the European Water Framework Directive was used. This data set includes 6 sampling locations at the Bavarian River Inn that were sampled in different seasons. Fish were caught according to a standardized national sampling protocol $[33,34]$. This standard included electrofishing from the boat or wading using an electrofishing generator with continuous voltage and a single anode by specifically trained personnel with in-depth training on correct species identification. All fish caught were assigned to species-specific size classes, and numbers were recorded, distinguishing juveniles, subadults, and adults [35].

\subsection{Physicochemical Habitat Characteristics and Vegetation}

To characterize structural and chemical habitat properties of the FPs, physicochemical habitat variables were recorded at the same time as the fish sampling was carried out (Tables 2-4). Habitat surface area (wetted area of ponds and fluvial habitats given in $\left.\mathrm{m}^{2}\right)$, water depth $(\mathrm{m})$, length of fluvial habitats $(\mathrm{m})$, width of fluvial habitats $(\mathrm{m})$, and current speed (Ott MF pro, Ott, Kempten, Germany) $5 \mathrm{~cm}$ above ground $(\mathrm{m} / \mathrm{s})$, as well as $5 \mathrm{~cm}$ below surface $(\mathrm{m} / \mathrm{s})$. The measurements were recorded at 9 measurement points distributed along three cross sections in each fluvial habitat and pond, respectively (Tables 2 and 3). Readings of electric conductance (EC, $\mu \mathrm{S} / \mathrm{cm}$, corrected to $20^{\circ} \mathrm{C}$ ), dissolved oxygen concentration $\left(\mathrm{O}_{2}, \mathrm{mg} / \mathrm{L}\right), \mathrm{pH}$ value $(\mathrm{pH})$, temperature $\left(\mathrm{T},{ }^{\circ} \mathrm{C}\right)$, and turbidity (Turb, NTU) were taken using the handheld devices Multi 3430, pH 3110, and pHotoFlex Turb (WTW, Weilheim, Germany), with three measurements per fluvial habitat or pond (Table 4). The relative composition of bed material was visually estimated in $10 \%$ steps according to the classification boulders (B), gravel $(\mathrm{G})$, sand (S), and fines $(\mathrm{F})$. The presence of bank vegetation (VEG), macrophytes (MA), and dead wood (DW) was documented in $5 \%$ steps (Tables 2 and 3, following Reference [19]).

\subsection{Data Analysis}

All multivariate analyses were computed using PRIMER v7 (Plymouth Marine Laboratory, Plymouth, UK). For all statistical analyses, significance was accepted at $p \leq 0.05$. To analyze differences in fish community composition, a catch per unit effort (CPUE) standardized to $10 \mathrm{~m}$ fished river length for the comparison of the Inn with FPs, and $10 \mathrm{~m}^{2}$ for multivariate comparisons between FPs, seasons, and FP habitats was calculated. Due to the different data structure of the water framework sampling conducted in the main stem of the River Inn and the FP (sampling of $30 \mathrm{~m}$ fluvial habitats and ponds), the resemblance matrix for the non-metric multidimensional scaling (nMDS) for the comparison River Inn versus FP was calculated based on pooled seasonal data, which is displayed in Figure 4A. For the comparisons of fish community composition within the FPs, a second resemblance matrix was created using the full data resolution of all fluvial habitats and ponds during different seasons (spring, summer, and late fall; Figure 4B). Additionally, the bubble-function in PRIMERv7 was used to display the contribution of important target species for restoration, such as B. barbus, C. nasus, $H$. hucho, and T. thymallus ( $\mathrm{N}=$ number of individuals), to the 
fish community composition (Figure 4C). To identify relations between fish community data and environmental data, biota-environmental stepwise matching (BEST) analyses was conducted, using the biota-environmental matching (Bio-Env) method to identify the significant relationships between fish community composition and the assessed physicochemical habitat variables within fluvial habitats and ponds (Bio-Env; Reference [36]). Abiotic habitat variables were plotted in the nMDS using the overlay function in PRIMER (Figure 4B). To test for significant differences between fish community composition of the River Inn and FPs, between FP Gars and FP Stammham, and between fluvial habitats and ponds in different seasons, one-way analysis of similarities (ANOSIM) based on BrayCurtis similarities [37] calculated from species abundance and species length-frequency data [38] were computed. If variables amongst samples happened to be entirely zero, a zero-adjusted Bray-Curtis coefficient, including a virtual dummy variable being one for all objects, was used as suggested by Clarke et al. [39]. To identify the most common and steadily occurring species in the River Inn, among the FPs, FS, and ponds and at different seasons, a one-way Similarity Percentage Analysis (SIMPER; Reference [36]) was computed. Average dissimilarity values for the abundance of species for the respective comparisons Inn versus FPs, FP Gars versus FP Stammham, FS versus ponds, and the comparisons among different seasons were calculated (Table 5).

Table 4. Abiotic habitat variables measured in the two fish passes Gars and Stammham throughout the study period. Values represent Scheme 2. $\mathrm{O}_{2}=$ dissolved oxygen, $\mathrm{EC}=$ electric conductance, $\mathrm{pH}$, and turbidity. NA = not available.

\begin{tabular}{|c|c|c|c|c|c|c|c|c|c|c|}
\hline & \multicolumn{5}{|c|}{ Gars Fluvial Habitats } & \multicolumn{5}{|c|}{ Gars Ponds } \\
\hline & $\mathrm{T}\left[{ }^{\circ} \mathrm{C}\right]$ & $\underset{\left[\mathrm{mgl}^{-1}\right]}{\mathrm{O}_{2}}$ & $\underset{\left[\mu \mathrm{scm}^{-1}\right]}{\mathrm{EC}}$ & $\mathrm{pH}$ & $\begin{array}{c}\text { Turbidity } \\
\text { [NTU] }\end{array}$ & $\mathrm{T}\left[{ }^{\circ} \mathrm{C}\right]$ & $\underset{\left[\mathrm{mgl}^{-1}\right]}{\mathrm{O}_{2}}$ & $\underset{\left[\mu \mathrm{sCm}^{-1}\right]}{\mathrm{EC}}$ & $\mathrm{pH}$ & $\begin{array}{c}\text { Turbidity } \\
\text { [NTU] }\end{array}$ \\
\hline \multirow[t]{2}{*}{ March } & 6.4 & 12.1 & 385 & 8.2 & 16.3 & 7.6 & 14.2 & 358 & 8.5 & 12.3 \\
\hline & $6.4-6.5$ & $\begin{array}{l}11.8- \\
12.8\end{array}$ & $380-388$ & $8.2-8.3$ & $\begin{array}{l}12.1- \\
24.8\end{array}$ & $6.6-8.4$ & $\begin{array}{l}11.0- \\
16.6\end{array}$ & $352-416$ & $8.0-8.9$ & $2.6-19.6$ \\
\hline \multirow[t]{2}{*}{ May } & 9.1 & 11.1 & 276 & 8.3 & 36.0 & 10.7 & 12.3 & 272 & 8.5 & 19.1 \\
\hline & $8.6-9.4$ & $\begin{array}{l}11.0- \\
11.1\end{array}$ & $273-279$ & $8.2-8.5$ & $\begin{array}{l}28.5- \\
45.1\end{array}$ & $8.8-12.5$ & $\begin{array}{l}11.0- \\
15.3\end{array}$ & $263-280$ & $8.3-8.9$ & $6.9-35.3$ \\
\hline \multirow[t]{2}{*}{ June } & 13.8 & 10.2 & 194 & NA & 117.0 & 16.1 & 10.4 & 191 & NA & 60.5 \\
\hline & $\begin{array}{l}13.6- \\
14.1\end{array}$ & $\begin{array}{l}10.1- \\
10.4\end{array}$ & 195-199 & NA & $\begin{array}{l}101.6- \\
134.9\end{array}$ & $\begin{array}{l}14.3- \\
20.5\end{array}$ & $9.3-11.2$ & 169-197 & NA & $\begin{array}{l}12.5- \\
113.0\end{array}$ \\
\hline \multirow[t]{2}{*}{ August } & 18.6 & 9.1 & 224 & 8.0 & 167.7 & 22.0 & 10.0 & 208 & 8.5 & 78 \\
\hline & $\begin{array}{l}18.2- \\
19.1\end{array}$ & $7.8-9.7$ & $218-231$ & $7.9-8.1$ & $\begin{array}{l}139.0- \\
215.2\end{array}$ & $\begin{array}{l}18.9- \\
27.2\end{array}$ & $7.2-13.3$ & $150-225$ & $7.6-9.7$ & $\begin{array}{r}5.5- \\
141.8\end{array}$ \\
\hline \multirow[t]{2}{*}{ October } & 11.8 & 10.9 & 330 & 8.1 & 16.4 & 13.3 & 11.6 & 303 & 8.4 & 7.3 \\
\hline & $\begin{array}{l}11.5- \\
12.1\end{array}$ & $\begin{array}{l}10.3- \\
11.1\end{array}$ & $327-333$ & $7.9-8.2$ & $\begin{array}{l}11.1- \\
23.2\end{array}$ & $\begin{array}{l}12.7- \\
14.8\end{array}$ & $4.0-18.3$ & $241-386$ & $7.8-8.9$ & $2.7-17.3$ \\
\hline \multirow[t]{4}{*}{ December } & 4.8 & 11.7 & 388 & 8.1 & 15.7 & 3.0 & 13.4 & 336 & 8.6 & 10.1 \\
\hline & $4.7-4.9$ & $\begin{array}{l}11.1- \\
11.9\end{array}$ & $359-393$ & $7.9-8.2$ & $\begin{array}{l}10.1- \\
25.6\end{array}$ & $2.4-3.4$ & $\begin{array}{l}10.3- \\
16.2\end{array}$ & $275-362$ & $8.1-8.8$ & $0.5-22.9$ \\
\hline & & Stamm & am Fluvial & abitats & & & & nmham Poi & & \\
\hline & $\mathrm{T}\left[{ }^{\circ} \mathrm{C}\right]$ & $\begin{array}{c}\mathrm{O}_{2} \\
{\left[\mathrm{mgl}^{-1}\right]}\end{array}$ & $\underset{\left[\mu \mathrm{scm}^{-1}\right]}{\mathrm{EC}}$ & $\mathrm{pH}$ & $\begin{array}{c}\text { Turbidity } \\
\text { [NTU] }\end{array}$ & $\mathrm{T}\left[{ }^{\circ} \mathrm{C}\right]$ & $\begin{array}{c}\mathrm{O}_{2} \\
{\left[\mathrm{mg}^{-1}\right]}\end{array}$ & $\underset{\left[\mu \mathrm{scm}^{-1}\right]}{\mathrm{EC}}$ & $\mathrm{pH}$ & $\begin{array}{c}\text { Turbidity } \\
\text { [NTU] }\end{array}$ \\
\hline \multirow[t]{2}{*}{ March } & 7.0 & 11.2 & 425 & 8.0 & 16.0 & 7.4 & 7.7 & 425 & 7.8 & 3.5 \\
\hline & $6.6-7.1$ & $\begin{array}{l}11.0- \\
11.7\end{array}$ & 379-441 & 7.9-8.1 & $\begin{array}{l}10.6- \\
26.3\end{array}$ & $6.7-9.1$ & $1.2-13.0$ & $405-442$ & $6.7-8.5$ & $0.2-8.7$ \\
\hline \multirow[t]{2}{*}{ May } & 9.0 & 11.0 & 327 & 8.2 & 20.3 & 13.3 & 10.7 & 369 & 8.2 & 4.4 \\
\hline & $8.7-9.3$ & $\begin{array}{l}10.0- \\
11.3\end{array}$ & $304-365$ & $8.1-8.3$ & $\begin{array}{l}12.5- \\
27.7\end{array}$ & $9.3-19.6$ & $3.8-16.9$ & $319-418$ & $7.8-8.6$ & $1.8-11.4$ \\
\hline \multirow[t]{2}{*}{ June } & 14.6 & 9.9 & 253 & NA & 72.2 & 17.9 & 9.3 & 295 & NA & 25.8 \\
\hline & $\begin{array}{l}14.0- \\
14.8\end{array}$ & $9.7-10.1$ & 234-288 & NA & $\begin{array}{l}52.0- \\
90.3\end{array}$ & $\begin{array}{l}12.5- \\
24.5\end{array}$ & $4.4-13.1$ & $207-354$ & NA & $2.2-74.6$ \\
\hline \multirow[t]{2}{*}{ August } & 19.1 & 8.8 & 264 & 8.0 & 79.9 & 23.3 & 12.7 & 269 & 8.6 & 27.9 \\
\hline & $\begin{array}{l}18.3- \\
19.7\end{array}$ & $7.7-9.0$ & $245-295$ & $7.9-8.9$ & $\begin{array}{l}59.4- \\
107.0\end{array}$ & $\begin{array}{l}15.1- \\
30.0\end{array}$ & $4.5-18.4$ & $203-328$ & $8.0-9.5$ & $\begin{array}{c}1.9- \\
128.7\end{array}$ \\
\hline \multirow[t]{2}{*}{ October } & 12.8 & 10.3 & 355 & 8.1 & 9.9 & 13.9 & 8.1 & 335 & 8.0 & 3.2 \\
\hline & $\begin{array}{l}12.3- \\
13.2\end{array}$ & $9.5-11.0$ & $342-379$ & $7.9-8.2$ & $5.8-17.1$ & $\begin{array}{l}11.6- \\
15.8\end{array}$ & $1.8-10.9$ & $297-376$ & $7.5-8.5$ & $1.1-5.6$ \\
\hline \multirow[t]{2}{*}{ December } & 5.1 & 11.2 & 407 & 8.1 & 17.1 & 5.6 & 8.0 & 388 & 7.9 & 7.4 \\
\hline & $4.7-6.1$ & $\begin{array}{l}10.5- \\
11.8 \\
\end{array}$ & $400-423$ & $8.0-8.1$ & $7.9-29.2$ & $4.4-9.8$ & $2.6-11.0$ & $373-421$ & $7.6-8.2$ & $0.3-20.9$ \\
\hline
\end{tabular}



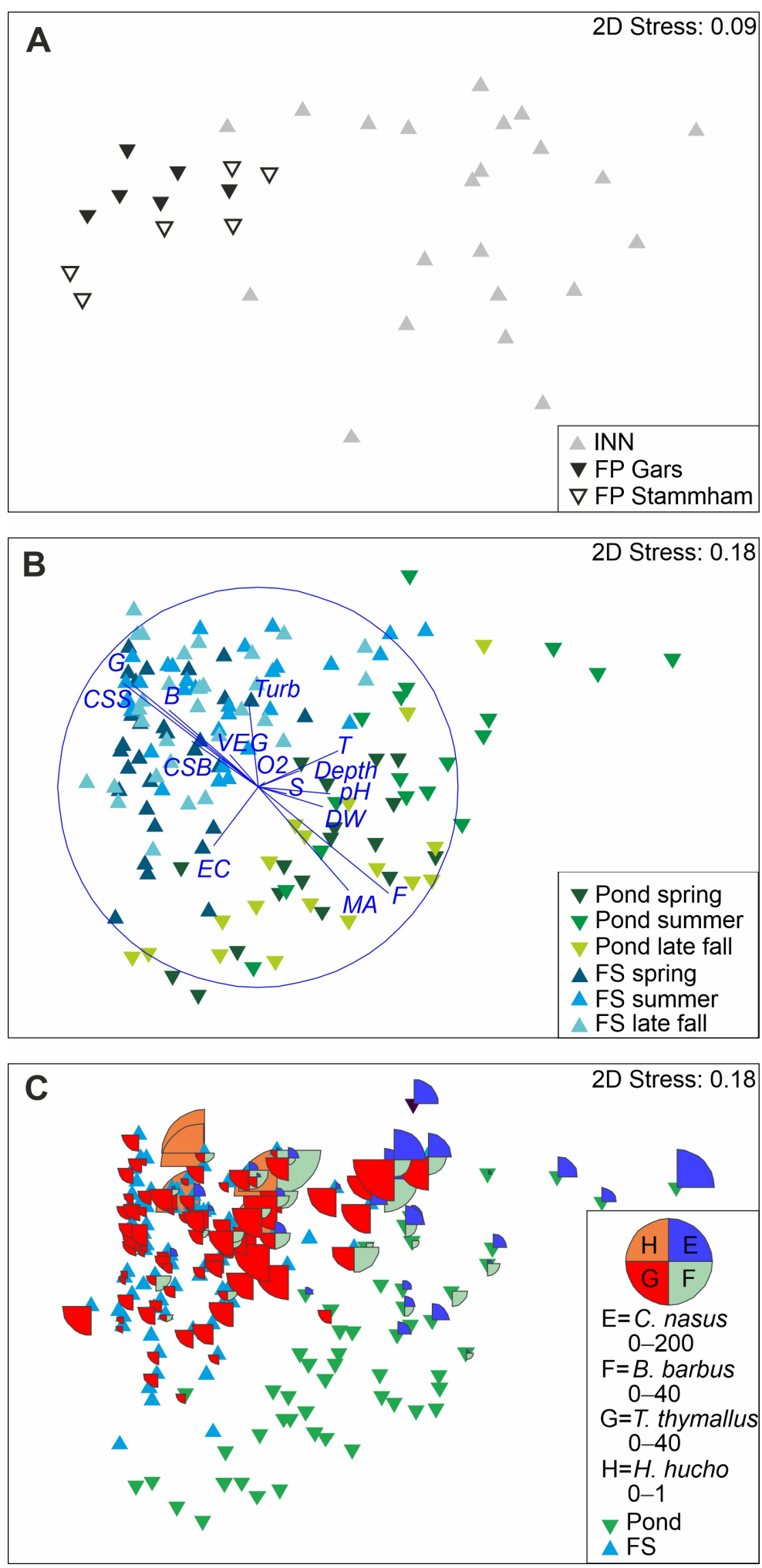

Figure 4. Non-metric multidimensional scaling (nMDS) comprising comparisons of fish community composition based on catch per unit effort (CPUE) abundance data. (A) The River Inn and the fish passes $(\mathrm{Inn}=$ grey triangles, black triangles, = fish pass Gars, open black triangles = fish pass Stammham), (B) comparison of the fish community composition in ponds (green triangles) versus fluvial habitats (blue triangles), and (C) proportion of target species in ponds or fluvial habitats displayed as pie size. Green triangles (ponds) and blue triangles (fluvial habitats). C. nasus = Chondrostoma nasus, B. barbus = Barbus barbus, T. thymallus = Thymallus thymallus, and H. hucho= Hucho hucho. Abbreviation of habitat variables refer to Tables $2-4$. Turb = turbidity in NTU. 2D-Stress = stress value after Kruskal. 
Table 5. Results of one-way analysis of similarities (ANOSIM) and Similarity Percentage Analysis (SIMPER) concerning pairwise comparisons of the two fish passes, different seasons, and between fluvial habitats and ponds. AVDIS = average dissimilarity, NA = not detected by SIMPER. FP = fish pass, FS = fluvial habitats, $\mathrm{PO}=$ floodplain ponds. For abbreviations of species, refer to Table 6.

\begin{tabular}{|c|c|c|c|c|c|c|c|c|c|c|c|c|c|c|c|c|}
\hline \multirow[b]{2}{*}{ Comparisons } & \multicolumn{3}{|c|}{ ANOSIM } & \multicolumn{13}{|c|}{ SIMPER } \\
\hline & $\begin{array}{c}\text { R- } \\
\text { Value }\end{array}$ & $\begin{array}{c}p- \\
\text { Value }\end{array}$ & df & AVDIS & Bleak & Stonel & Barbel & Bullh & Nase & Stickle & Dace & $\begin{array}{c}\text { Lamprey } \\
\text { Larvae }\end{array}$ & Stomo & Bitter & Chub & Grayl \\
\hline FP-River Inn & 0.482 & $<0.001$ & 1 & 88.42 & $\begin{array}{l}9.44- \\
17.89\end{array}$ & NA & NA & $\begin{array}{l}47.46- \\
3.02\end{array}$ & NA & $\begin{array}{l}52.95- \\
0.30\end{array}$ & NA & NA & NA & NA & $\begin{array}{l}76.57- \\
7.13\end{array}$ & $\begin{array}{c}12.96- \\
0.71\end{array}$ \\
\hline $\begin{array}{l}\text { FP Gars-FP } \\
\text { Stammham }\end{array}$ & 0.07 & $<0.001$ & 1 & 83.28 & NA & NA & NA & $\begin{array}{c}20.15- \\
6.31\end{array}$ & $\begin{array}{l}3.36- \\
7.46\end{array}$ & $\begin{array}{l}5.71- \\
25.49\end{array}$ & NA & $0.00-6.04$ & NA & NA & $\begin{array}{l}18.71- \\
25.36\end{array}$ & $\begin{array}{l}2.26- \\
5.29\end{array}$ \\
\hline $\begin{array}{l}\text { FP spring-FP } \\
\text { summer }\end{array}$ & 0.134 & $<0.001$ & 2 & 82.97 & $\begin{array}{l}7.85- \\
13.92\end{array}$ & NA & NA & NA & $\begin{array}{c}1.17 \\
-14.38\end{array}$ & $\begin{array}{l}3.17- \\
40.40\end{array}$ & NA & $2.46-5.44$ & NA & NA & $\begin{array}{l}2.73- \\
58.19\end{array}$ & $\begin{array}{l}1.69- \\
5.15\end{array}$ \\
\hline $\begin{array}{l}\text { FP summer-FP } \\
\text { late fall }\end{array}$ & 0.051 & $<0.01$ & 2 & 81.22 & NA & NA & NA & $\begin{array}{l}13.92- \\
16.19\end{array}$ & $\begin{array}{c}14.38- \\
1.21\end{array}$ & $40.40-$ & $\begin{array}{l}6.83- \\
3.54\end{array}$ & NA & NA & NA & $\begin{array}{l}58.19- \\
6.02\end{array}$ & $\begin{array}{l}5.15- \\
4.88\end{array}$ \\
\hline $\begin{array}{l}\text { FP spring-FP } \\
\text { late fall }\end{array}$ & 0.023 & $>0.05$ & 2 & 82.31 & NA & NA & NA & $\begin{array}{l}7.85- \\
16.19\end{array}$ & NA & $\begin{array}{l}3.17- \\
5.71\end{array}$ & NA & $2.46-1.92$ & NA & NA & $\begin{array}{l}2.73- \\
6.02\end{array}$ & $\begin{array}{l}1.69- \\
4.88\end{array}$ \\
\hline FS-PO & 0.631 & $<0.001$ & 1 & 92.62 & NA & NA & NA & $\begin{array}{c}19.97- \\
0.46\end{array}$ & $\begin{array}{l}4.19- \\
7.91\end{array}$ & $\begin{array}{l}1.81- \\
40.78\end{array}$ & NA & NA & NA & NA & $\begin{array}{l}4.42- \\
52.13\end{array}$ & $\begin{array}{l}6.18- \\
0.11\end{array}$ \\
\hline $\begin{array}{l}\text { PO Gars-PO } \\
\text { Stammham }\end{array}$ & 0.122 & $<0.01$ & 3 & 84.05 & NA & $\begin{array}{l}3.70- \\
0.58\end{array}$ & NA & NA & $\begin{array}{l}6.23- \\
10.00\end{array}$ & $\begin{array}{l}12.53- \\
76.08\end{array}$ & $\begin{array}{l}7.63- \\
5.46\end{array}$ & NA & NA & NA & $\begin{array}{l}39.40- \\
68.04\end{array}$ & NA \\
\hline $\begin{array}{l}\text { FS Gars-FS } \\
\text { Stammham }\end{array}$ & 0.208 & $<0.001$ & 3 & 72.10 & NA & NA & NA & $\begin{array}{l}36.25- \\
9.11\end{array}$ & NA & NA & $\mathrm{NA}$ & $0.00-8.50$ & NA & NA & $\begin{array}{l}1.47- \\
6.39\end{array}$ & $\begin{array}{l}3.97- \\
7.65\end{array}$ \\
\hline PO Gars-FS Gars & 0.795 & $<0.001$ & 3 & 93.64 & NA & NA & NA & $\begin{array}{l}0.83- \\
36.25\end{array}$ & NA & $\begin{array}{c}12.53- \\
0.03\end{array}$ & NA & NA & NA & NA & $\begin{array}{c}39.40- \\
1.47\end{array}$ & NA \\
\hline $\begin{array}{c}\mathrm{PO} \\
\text { Stammham-FS } \\
\text { Stammham }\end{array}$ & 0.635 & $<0.001$ & 3 & 93.34 & NA & NA & NA & $\begin{array}{l}0.00- \\
9.11\end{array}$ & $\begin{array}{l}10.00- \\
6.33\end{array}$ & $\begin{array}{l}76.08- \\
3.00\end{array}$ & NA & $0.50-8.50$ & NA & $\begin{array}{l}9.42- \\
0.13\end{array}$ & $\begin{array}{c}68.04- \\
6.39\end{array}$ & $\begin{array}{l}0.00- \\
7.65\end{array}$ \\
\hline $\begin{array}{l}\text { FS spring-FS } \\
\text { summer }\end{array}$ & 0.181 & $<0.001$ & 5 & 71.43 & NA & NA & $\begin{array}{l}0.03- \\
4.30\end{array}$ & $\begin{array}{l}12.40- \\
21.77\end{array}$ & $\begin{array}{l}0.83- \\
9.83\end{array}$ & NA & NA & $3.83-8.60$ & NA & NA & $\begin{array}{l}0.97- \\
7.13\end{array}$ & $\begin{array}{l}2.50- \\
8.23\end{array}$ \\
\hline $\begin{array}{l}\text { FS summer-FS } \\
\text { late fall }\end{array}$ & 0.009 & $>0.05$ & 5 & 65.93 & $\begin{array}{l}3.30- \\
4.67\end{array}$ & NA & NA & $\begin{array}{l}21.77- \\
25.73\end{array}$ & $\begin{array}{l}9.83- \\
1.90\end{array}$ & NA & NA & $8.60-2.87$ & NA & NA & $\begin{array}{l}7.13- \\
5.17\end{array}$ & $\begin{array}{r}8.23 \\
-7.80\end{array}$ \\
\hline $\begin{array}{l}\text { FS spring-FS late } \\
\text { fall }\end{array}$ & 0.119 & $<0.001$ & 5 & 70.00 & NA & NA & NA & $\begin{array}{l}12.40- \\
25.73\end{array}$ & NA & NA & NA & $3.83-2.87$ & NA & NA & $\begin{array}{l}0.97- \\
5.17\end{array}$ & $\begin{array}{l}2.50- \\
7.80\end{array}$ \\
\hline $\begin{array}{l}\text { PO spring-PO } \\
\text { summer }\end{array}$ & 0.201 & $<0.001$ & 5 & 82.62 & NA & NA & NA & NA & $\begin{array}{l}1.72- \\
21.94\end{array}$ & $\begin{array}{c}8.06- \\
105.22\end{array}$ & NA & NA & NA & NA & $\begin{array}{c}5.67 \\
143.28\end{array}$ & NA \\
\hline $\begin{array}{l}\text { PO summer-PO } \\
\text { late fall }\end{array}$ & 0.207 & $<0.01$ & 5 & 85.21 & NA & NA & NA & NA & $\begin{array}{l}21.94- \\
0.06\end{array}$ & $\begin{array}{l}105.22- \\
9.06\end{array}$ & NA & NA & NA & NA & $\begin{array}{l}143.28- \\
7.44\end{array}$ & NA \\
\hline $\begin{array}{c}\mathrm{PO} \text { spring-PO } \\
\text { late fall }\end{array}$ & 0.017 & $>0.05$ & 5 & 81.34 & NA & $\begin{array}{l}2.56- \\
1.72\end{array}$ & NA & NA & NA & $\begin{array}{l}8.06- \\
9.06\end{array}$ & NA & NA & $\begin{array}{l}1.00- \\
1.33\end{array}$ & $\begin{array}{l}1.11- \\
2.50\end{array}$ & $\begin{array}{l}5.67- \\
7.44\end{array}$ & NA \\
\hline PO spring-FS & 0.699 & $<0.001$ & 5 & 92.69 & NA & $\begin{array}{l}2.56- \\
0.70\end{array}$ & NA & $\begin{array}{l}0.28- \\
12.40\end{array}$ & NA & $\begin{array}{l}8.06- \\
0.23\end{array}$ & NA & $0.17-3.83$ & NA & NA & $\begin{array}{l}5.67- \\
0.97\end{array}$ & NA \\
\hline $\begin{array}{l}\text { PO summer-FS } \\
\text { summer }\end{array}$ & 0.644 & $<0.001$ & 5 & 90.50 & NA & NA & NA & $\begin{array}{l}0.83- \\
21.77\end{array}$ & $\begin{array}{c}21.94- \\
9.83\end{array}$ & $\begin{array}{c}105.22- \\
1.50\end{array}$ & NA & NA & NA & NA & $\begin{array}{c}143.28- \\
7.13\end{array}$ & $\begin{array}{l}0.00- \\
8.23\end{array}$ \\
\hline $\begin{array}{l}\text { PO late fall-FS } \\
\text { late fall }\end{array}$ & 0.723 & $<0.001$ & 5 & 93.79 & NA & NA & NA & $\begin{array}{l}0.28- \\
25.73\end{array}$ & NA & $\begin{array}{l}9.06- \\
3.70\end{array}$ & $\begin{array}{l}5.83- \\
2.17\end{array}$ & NA & NA & NA & $\begin{array}{l}7.44- \\
5.17\end{array}$ & $\begin{array}{l}0.00- \\
7.80\end{array}$ \\
\hline
\end{tabular}

Numbers in bold indicate significant differences for pairwise comparisons.

To display species preferential use of FS or ponds, a scatter plot based on pooled species abundance data from FPs (data was $\log (X+1)$ transformed due to the highly unbalanced occurrence of species) was computed using the scatter plot function in PRIMERv7. To illustrate fish density of important or target species, cumulative bar plots for each month were displayed for FS and ponds, respectively. Additionally, length-frequency plots were computed for the most abundant species $S$. cephalus and the two target species of conservation $C$. nasus and T. thymallus.

Significant influences of abiotic parameters on fish species diversity in the ponds were assessed by computing a linear model (LM) as model assumptions regarding normal distribution of model residuals were met. Assumptions and model fit were assessed using the package "DHARMa" in R [40]. Species richness (numbers of species detected) as the response variable was linked to habitat variables described in Tables 2-4 as predictor variables. Data were visually checked for autocorrelation using autocorrelation (ACF) and partial autocorrelation (PACF) plots. To test whether possible temporal correlation of repeated sampling in the same habitat explained additional variability in the models, a linear mixed model (LMM) with the function "lmer" in the package "lme4" in R [41] was computed, including the sampling date (1-6) and site as random effects. Comparing models via akaike information criterion (AIC) values indicated no additional explanatory value to the model outcome when the random effects date and site were included. Hence, significances of main effects were tested in the initial model, excluding random effects, using an F test in the $\mathrm{R}$ "car" package [42].

\section{Results}

In the two fish passes 11,893 individuals of fish and lampreys were caught during the sampling survey. The 33 fish species detected belonged to nine families with Cyprinidae (55\% of all species) dominating, followed by Salmonidae (15\%) and Percidae (9\%). The 
Families Esocidae, Nemacheilidae, Cottidae, Cobitidae, and Gasterosteidae comprised only one species each (Table 6). Most abundant species were S. cephalus ( $27 \%$ of all individuals), followed by Gasterosteus aculeatus L. (20\%), C. gobio (15\%), C. nasus (7\%), T. thymallus (5\%), A. alburnus (4\%), Leuciscus leuciscus L. (4\%), R. rutilus (4\%), Barbatula barbatula L. (3\%), and Rhodeus amarus Bloch (2\%). All other species only contributed less than $1 \%$ to the individuals (Table 6).

Table 6. List of detected species and their abbreviations used. INN $=$ River Inn, FP $=$ fish pass, $\mathrm{CP}=$ current preference, $\mathrm{RL}=$ Red List Germany, RLB = Red List Bavaria [43], FFH = protected species according to Flora Fauna Habitat Direction AnnexII and AnnexV [44]. Please note that the number of individuals are given as total catch numbers, which were not standardized to the length of sampled area here.

\begin{tabular}{|c|c|c|c|c|c|c|c|c|c|c|c|}
\hline Species & $\begin{array}{l}\text { Common } \\
\text { Names }\end{array}$ & Abbreviation & INN & $\mathbf{F P}$ & Gars & Stammham & Family & $\mathrm{CP}$ & RL & RLB & FFH \\
\hline $\begin{array}{l}\text { Anguilla } \\
\text { anguilla }\end{array}$ & European eel & Eel & 225 & 0 & 0 & 0 & Anguillidae & indifferent & 3 & F & NL \\
\hline Abramis brama & $\begin{array}{l}\text { Freshwater } \\
\text { bream }\end{array}$ & Bream & 162 & 5 & 4 & 1 & Cyprinidae & indifferent & * & NL & NL \\
\hline $\begin{array}{l}\text { Alburnoides } \\
\text { bipunctatus }\end{array}$ & Spirlin & Spirlin & 85 & 19 & 7 & 12 & Cyprinidae & rheophil & V & 2 & NL \\
\hline $\begin{array}{l}\text { Alburnus } \\
\text { alburnus }\end{array}$ & Bleak & Bleak & 1830 & 434 & 26 & 408 & Cyprinidae & indifferent & * & V & NL \\
\hline Aspius aspius & Asp & Asp & 10 & 0 & 0 & 0 & Cyprinidae & indifferent & 3 & 3 & Annex II \\
\hline $\begin{array}{l}\text { Barbatula } \\
\text { barbatula }\end{array}$ & Stone loach & Stonel & 70 & 305 & 191 & 114 & Nemacheilidae & rheophil & * & V & NL \\
\hline $\begin{array}{l}\text { Barbus barbus } \\
\text { Blicca bjoerkna }\end{array}$ & $\begin{array}{c}\text { Barbel } \\
\text { White bream }\end{array}$ & $\begin{array}{c}\text { Barbel } \\
\text { Whitebr }\end{array}$ & 112 & $\begin{array}{c}152 \\
1\end{array}$ & $\begin{array}{c}28 \\
0\end{array}$ & $\begin{array}{c}124 \\
1\end{array}$ & $\begin{array}{l}\text { Cyprinidae } \\
\text { Cyprinidae }\end{array}$ & $\begin{array}{l}\text { rheophil } \\
\text { indifferent }\end{array}$ & * & $\begin{array}{l}3 \\
\mathrm{NL}\end{array}$ & $\begin{array}{c}\text { Annex V } \\
\text { NL }\end{array}$ \\
\hline $\begin{array}{l}\text { Carassius } \\
\text { gibelio }\end{array}$ & Prussian carp & Cruci & 1 & 2 & 0 & 2 & Cyprinidae & indifferent & * & NL & NL \\
\hline $\begin{array}{c}\text { Chondrostoma } \\
\text { nasus }\end{array}$ & Common nase & Nase & 211 & 786 & 222 & 564 & Cyprinidae & rheophil & V & 2 & NL \\
\hline Cottus gobio & Bullhead & Bullh & 507 & 1822 & 1330 & 492 & Cottidae & rheophil & $*$ & $\mathrm{~V}$ & Annex II \\
\hline $\begin{array}{c}\text { Cyprinus carpio } \\
\text { Esox lucius }\end{array}$ & $\begin{array}{l}\text { Common carp } \\
\text { Northern pike }\end{array}$ & $\begin{array}{l}\text { Carp } \\
\text { Pike }\end{array}$ & $\begin{array}{c}7 \\
110\end{array}$ & $\begin{array}{c}5 \\
32\end{array}$ & $\begin{array}{l}5 \\
4\end{array}$ & $\begin{array}{c}0 \\
28\end{array}$ & $\begin{array}{l}\text { Cyprinidae } \\
\text { Esocidae }\end{array}$ & $\begin{array}{l}\text { indifferent } \\
\text { indifferent }\end{array}$ & * & $\begin{array}{l}\mathrm{NL} \\
\mathrm{NL}\end{array}$ & $\begin{array}{l}\text { NL } \\
\text { NL }\end{array}$ \\
\hline $\begin{array}{l}\text { Gasterosteus } \\
\text { aculeatus }\end{array}$ & $\begin{array}{c}\text { Three-spined } \\
\text { stickleback }\end{array}$ & Stickle & 15 & 2365 & $\begin{array}{c}\mathbf{T} \\
377\end{array}$ & 1988 & Gasterosteidae & limnophil & * & $\mathrm{V}$ & $\mathrm{NL}$ \\
\hline Gobio gobio & Gudgeon & Gudge & 23 & 125 & 0 & 125 & Cyprinidae & rheophil & * & V & NL \\
\hline $\begin{array}{c}\text { Gymnocephalus } \\
\text { cernua }\end{array}$ & Ruffe & Ruffe & 19 & 0 & 0 & 0 & Percidae & indifferent & * & V & NL \\
\hline $\begin{array}{l}\text { Hucho hucho } \\
\text { NA }\end{array}$ & $\begin{array}{l}\text { Huchen } \\
\text { Brook lamprey }\end{array}$ & $\begin{array}{l}\text { Huchen } \\
\text { Blamp }\end{array}$ & $\begin{array}{l}22 \\
20\end{array}$ & $\begin{array}{c}6 \\
532\end{array}$ & $\begin{array}{c}3 \\
15\end{array}$ & $\begin{array}{c}3 \\
517\end{array}$ & $\begin{array}{l}\text { Salmonidae } \\
\text { Petromyzontidae }\end{array}$ & $\begin{array}{l}\text { rheophil } \\
\text { rheophil }\end{array}$ & 2 & $\begin{array}{l}3 \\
1\end{array}$ & $\begin{array}{l}\text { Annex II } \\
\text { Annex II }\end{array}$ \\
\hline $\begin{array}{l}\text { Lepomis } \\
\text { gibbosus }\end{array}$ & Pumpkinseed & Pumpk & 0 & 9 & 2 & 7 & Percidae & indifferent & * & $\mathrm{NL}$ & NL \\
\hline $\begin{array}{l}\text { Leucaspius } \\
\text { delineatus }\end{array}$ & Belica & Belica & 0 & 1 & 0 & 1 & Cyprinidae & limnophil & * & 3 & NL \\
\hline Leuciscus idus & Ide & Ide & 26 & 0 & 0 & 0 & Cyprinidae & indifferent & 3 & $\mathrm{~V}$ & NL \\
\hline $\begin{array}{l}\text { Leuciscus } \\
\text { leuciscus }\end{array}$ & Common dace & Dace & 230 & 506 & 246 & 260 & Cyprinidae & indifferent & * & V & NL \\
\hline Lota lota & Burbot & Burb & 235 & 2 & 2 & 0 & Lotidae & indifferent & $\mathrm{V}$ & 2 & NL \\
\hline $\begin{array}{l}\text { Misgurnus an- } \\
\text { guillicaudatus }\end{array}$ & Pond loach & Pondl & 0 & 2 & 2 & 0 & Cobitidae & limnophil & $\diamond$ & $\mathrm{NL}$ & NL \\
\hline $\begin{array}{c}\text { Oncorhynchus } \\
\text { mykiss }\end{array}$ & Rainbow trout & Raintr & 110 & 23 & 14 & 9 & Salmonidae & rheophil & $\diamond$ & $\mathrm{NL}$ & NL \\
\hline Perca fluviatilis & $\begin{array}{l}\text { European } \\
\text { perch }\end{array}$ & Perch & 89 & 76 & 29 & 47 & Percidae & indifferent & * & NL & NL \\
\hline $\begin{array}{l}\text { Phoxinus } \\
\text { phoxinus }\end{array}$ & $\begin{array}{l}\text { Eurasian } \\
\text { minnow }\end{array}$ & Minnow & 28 & 6 & 0 & 6 & Cyprinidae & indifferent & * & 3 & NL \\
\hline $\begin{array}{c}\text { Pseudorasbora } \\
\text { parva }\end{array}$ & Stone moroko & Stomo & 4 & 44 & 43 & 1 & Cyprinidae & indifferent & $\diamond$ & NL & NL \\
\hline $\begin{array}{l}\text { Rhodeus } \\
\text { amarus }\end{array}$ & $\begin{array}{l}\text { European } \\
\text { bitterling }\end{array}$ & Bitter & 5 & 233 & 0 & 233 & Cyprinidae & limnophil & * & 2 & Annex II \\
\hline $\begin{array}{c}\text { Romanogobio } \\
\text { vladykovi }\end{array}$ & Gudgeon & Wgudge & 15 & 0 & 0 & 0 & Cyprinidae & rheophil & 2 & 2 & Annex II \\
\hline Rutilus rutilus & Roach & Roach & 267 & 526 & 44 & 482 & Cyprinidae & indifferent & $*$ & $\mathrm{NL}$ & NL \\
\hline $\begin{array}{c}\text { Salmo trutta } \\
\text { fario }\end{array}$ & Brown trout & Browntr & 331 & 77 & 16 & 61 & Salmonidae & rheophil & * & V & NL \\
\hline $\begin{array}{l}\text { Salvelinus } \\
\text { fontinalis }\end{array}$ & Brook trout & Brooktr & 4 & 1 & 1 & 0 & Salmonidae & rheophil & $\diamond$ & NL & NL \\
\hline $\begin{array}{c}\text { Sander } \\
\text { lucioperca }\end{array}$ & Pike-pearch & Pikepe & 12 & 1 & 0 & 1 & Percidae & indifferent & * & NL & NL \\
\hline $\begin{array}{l}\text { Scardinius ery- } \\
\text { throphthalmus }\end{array}$ & Rudd & Rudd & 4 & 2 & 1 & 1 & Cyprinidae & limnophil & * & $\mathrm{NL}$ & NL \\
\hline Silurus glanis & Wels catfish & Wels & 3 & 0 & 0 & 0 & Siluridae & indifferent & 2 & $\mathrm{~V}$ & NL \\
\hline $\begin{array}{l}\text { Squalius } \\
\text { cephalus }\end{array}$ & Chub & Chub & 837 & 3213 & 1235 & 1978 & Cyprinidae & indifferent & * & $\mathrm{NL}$ & NL \\
\hline $\begin{array}{l}\text { Thymallus } \\
\text { thymallus }\end{array}$ & Grayling & Grayl & 238 & 562 & 149 & 413 & Salmonidae & rheophil & 2 & 2 & Annex V \\
\hline Tinca tinca & Tench & Tench & 1 & 29 & 6 & 23 & Cyprinidae & limnophil & $*$ & NL & NL \\
\hline
\end{tabular}

Fish community composition in the fish passes based on pooled data over all sampling time points differed significantly from the River Inn (Figure 4, ANOSIM, $\mathrm{R}=0.48, p<0.001$ ). According to SIMPER analysis, these differences were mainly attributed to the higher 
abundance of C. gobio, S. cephalus, G. aculeatus, T. thymallus, and Petromyzontidae in the fish passes and higher abundance of A. alburnus in the main river (Table 5). Species richness in the River Inn was higher (40 species) than in the fish passes with six more species being caught. These were mainly ubiquitous species except for the Danube-endemic rheophilic specialist Romanogobio vladykovi Fang. Three additional species (Lepomis gibbosus L., Misgurnus bipartitus Dybowski, and Leucaspius delineates Heckel) were exclusively caught in the fish passes (Table 6).

The community composition of the FP Gars differed significantly from the FP Stammham; however, the $\mathrm{R}$-value was very low for this comparison (ANOSIM, $\mathrm{R}=0.07, p<0.001$ ). According to SIMPER, the same set of species was identified as for the comparison Inn and FP (Table 5), with C. gobio being 3-fold more abundant in Gars and S. cephalus, G. aculeatus, T. thymallus, and Petromyzontidae being more abundant in Stammham. Throughout the year, fish community composition in FP changed significantly (ANOSIM, $\mathrm{R}=0.058, p<0.001$ ) between spring (March and May), summer (June and August), and late fall (October and December) (Figure 4, Table 5). For example, SIMPER detected that species, such as C. gobio, increased in abundance from spring to late fall, whilst S. cephalus, G. aculeatus, and C. nasus reached their abundance peak in summer. T. thymallus was also detected with highest densities in summer, but, in contrast to the other species, it was still present in high abundances in the FP during late fall.

Fluvial habitats of the fish passes differed significantly from ponds in community composition (Figure 4, ANOSIM, $\mathrm{R}=0.631, p<0.001$ ). BEST analysis detected macrophyte coverage, gravel, boulders, temperature and current speed at the surface as variables best fitting the ordination of abundance data in the nMDS $\left(\mathrm{R}^{2}=0.74\right.$, Figure 4$)$. There was a higher proportion of macrophytes and a higher water temperature in ponds, and more gravel, boulders and higher current speed in fluvial habitats. According to SIMPER, differences in fish community structure among habitat types were mainly attributed to higher abundances of T. thymallus (56-fold), C. gobio (40-fold), C. nasus (1.9-fold) in fluvial habitats, and G. aculeatus (22-fold) and S. cephalus (12-fold) in ponds (Table 5). Five stagnophilic or indifferent species, such as L. gibbosus, Scardinius erythrophthalmus L., M. bipartitus, Cyprinus carpio L., and Blicca bjoerkna L., were exclusively detected in ponds (overall number of species here 26), whilst 6 mostly rheophilic species (e.g., Salvelinus fontinalis Mitchill, Gobio gobio L., O. mykiss, H. Hucho) were exclusively detected in fluvial habitats (28 species) of the FP (Figure 7, Table 6). C. gobio (99\% individuals), S. trutta (99\%), T. thymallus (99\%), B. barbus (95\%), and Petromyzontidae (97\%) were almost exclusively detected in the fluvial habitats, and $R$. amarus, as well as Pseudorasbora parva Temmink \& Schlegel, almost exclusively in ponds (Figure 7 , Table 6).

However, it has to be noted that the abundance of species in fluvial habitats or ponds was strongly governed by seasonal effects (Figure 5). This was particularly true for species that are known to switch habitats during different stages of their life cycle, such as $C$. nasus. This species was very abundant in ponds during summer and disappeared almost entirely from this habitat in late fall (Figures 5 and 6). In contrast, other species, like $S$. cephalus, used ponds and fluvial habitats equally throughout the year in almost all size classes (Figure 6), whilst R. amarus and P. parva only used ponds comprising a population structure, including all size classes. In contrast, all size classes of the reophilic species $T$. thymallus were exclusively found in fluvial habitats. LM detected that species richness in the ponds was significantly influenced by the factors macrophyte coverage $(p<0.05)$, deadwood coverage $(p<0.01)$ and water depth $(p<0.05)$. For all detections of significances, the relationship between the variable and the response species richness was not linear with highest species diversity for macrophyte coverage at $75 \%$, deadwood coverage between $5 \%$ and $10 \%$, and water depth about $0.5 \mathrm{~m}$ to $1.0 \mathrm{~m}$. 

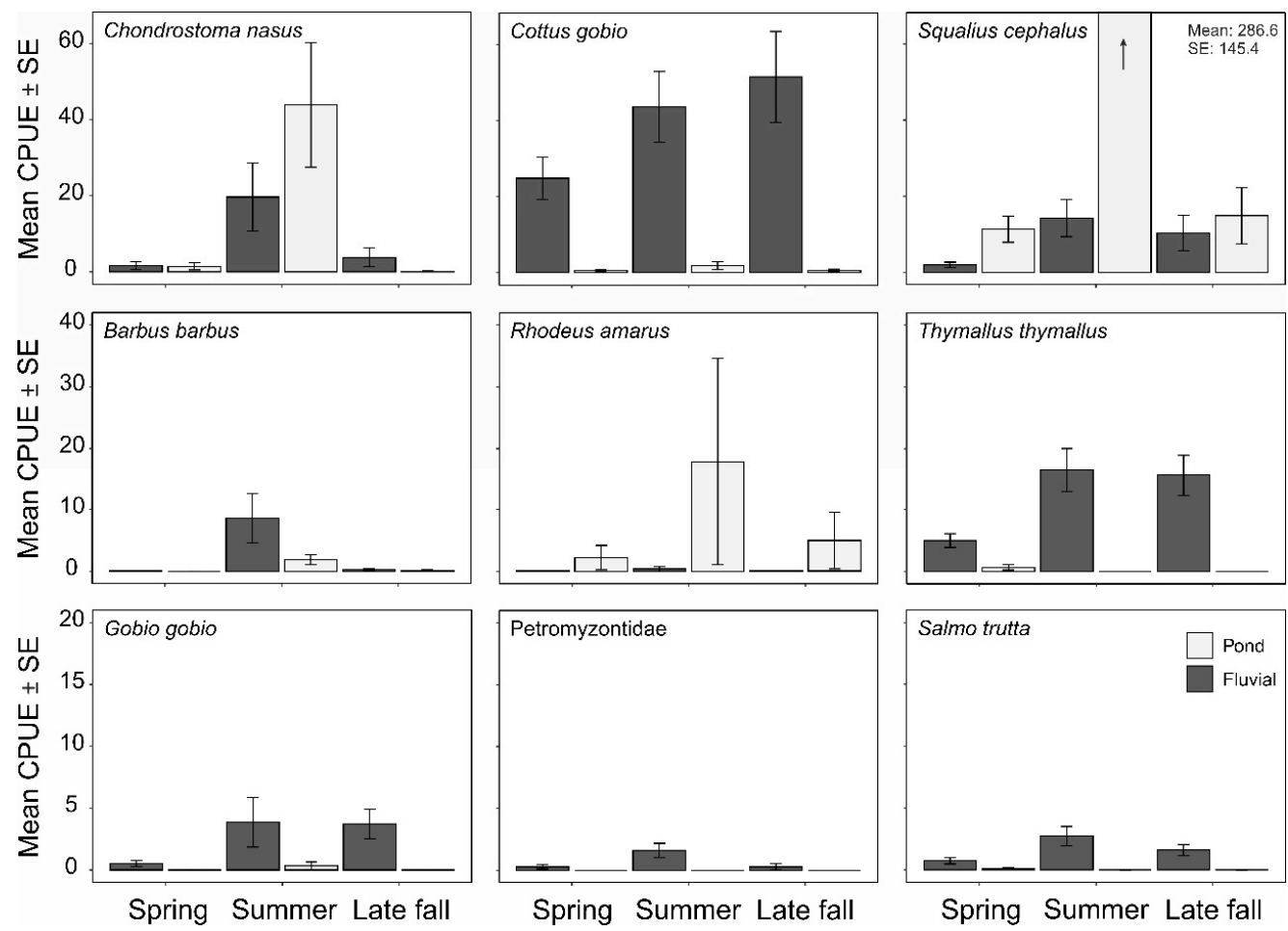

Figure 5. Seasonal distribution of the mean catch per unit effort \pm standard error (CPUE \pm SE) for selected target species of conservation. Different shades of grey indicate the habitat types 'pond ' (light grey) and 'fluvial habitat' (dark grey). Outlier is marked with an arrow. Note the different scaling of the y-axis.

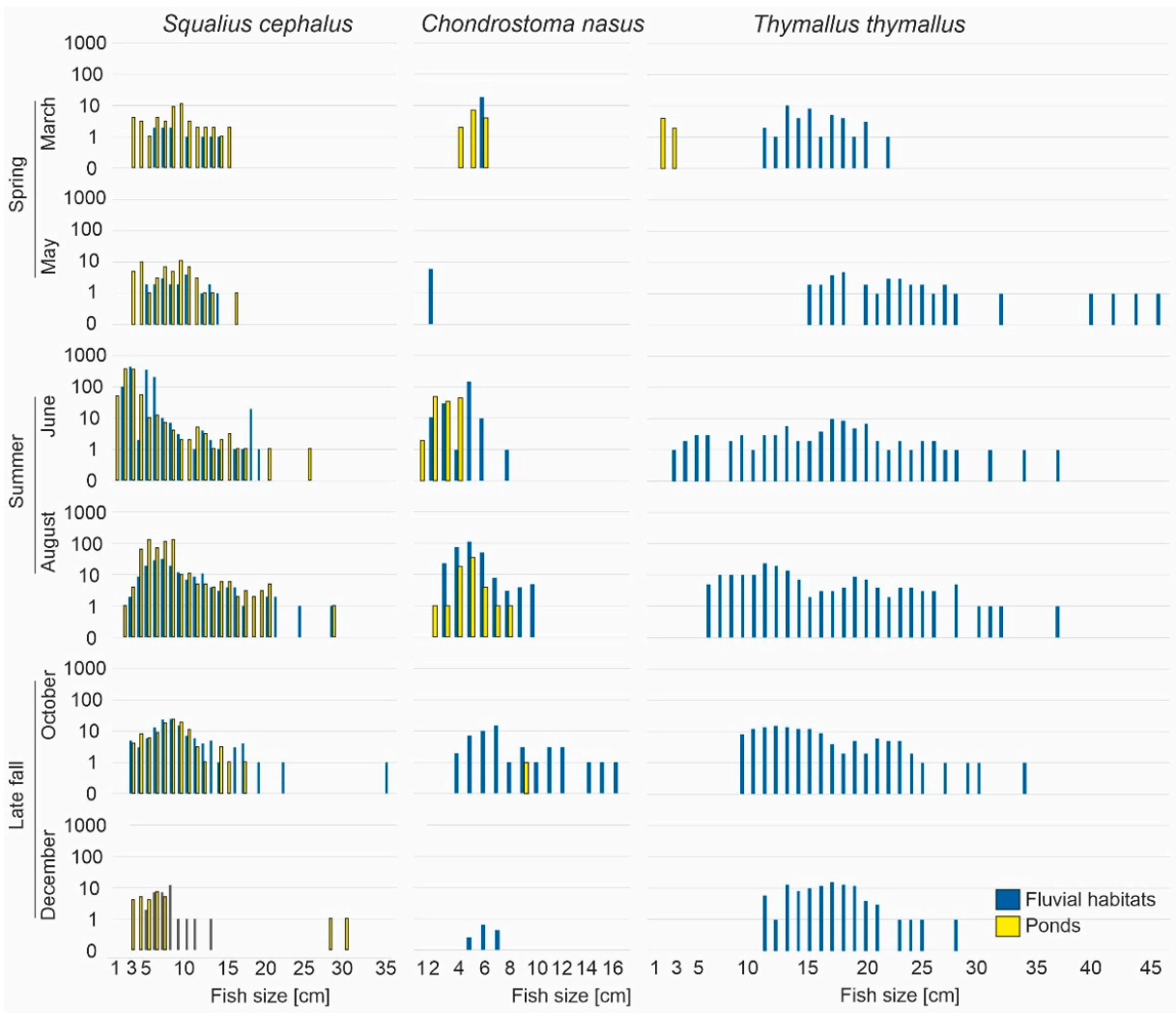

Figure 6. Length-frequency-distribution of Squalius cephalus, the most persistent fish species, as well as two other species with high conservation and indicator value, Thymallus thymallus and Chondrostoma nasus, in fluvial habitats (blue bars) and ponds (yellow bars), for the different sampling months between March and December and the seasons spring, summer, and late fall. Bars represent the sum of caught individuals. 


\section{Discussion}

The findings of this study confirm the positive contribution of diverse habitats within constructed fish passes to the fish diversity of the River Inn. Fish diversity within the fish passes (33 species) largely resembled the species inventory of the River Inn (36 species), except for some rare species, such as R. vladykovi, that also occur at low densities in the main river [30]. Still, there were major differences in abundances of species between the fish passes and the main river.

As demonstrated herein, nature- like fish passes of high habitat diversity, comprising both fluvial habitats and stagnant waters, can at least partially contribute to the restoration of the formerly present habitat mosaic in this alpine river. In such large degraded alpine systems as the River Inn, the restoration of river dynamic processes, which govern high habitat and species diversity, is almost impossible due to the many restrictions in densely populated areas. From a fish conservation point of view, this makes near-natural watercourses that can at least partially fulfil these functions most valuable.

As expected, fluvial habitats within the fish passes comprised a rheophilic-dominated fish community, whilst ponds were largely dominated by stagnophilic fish species, or by species with indifferent current preferences. Consequently, both habitats contributed to a high species diversity in that system. However, it has to be considered that the fish community sampling in these small structures within a fish pass is likely more effective than in the larger main stem [46], potentially resulting in a bias towards an underestimation of species richness in the River Inn. This is also in compliance with the findings from Mollenhauer et al. [47], who demonstrated that fish detection probability can be affected by water depth and turbidity.

The populations of species, such as T. thymallus, B. barbus, and C. nasus, are under pressure in almost all rivers across their distribution range, particularly in the densest populated areas of central Europe, with their names on national and international red lists alike [43]. This makes them prominent target species for conservation. The fact that $T$. thymallus was detected in all size classes and that $C$. nasus and B. barbus were present in their most sensitive small size classes indicate that fluvial habitats and ponds in nature-like fish passes can provide important juvenile habitats, which are scarce in the main river. This should encourage river managers to follow the pathway of creating additional fluvial habitats and small ponds adjacent to the heavily modified and exploited main channel of rivers such as the Inn. The presence of early life stages of those target species and their seasonal habitat use, as detected herein for C. nasus, indicates that, besides habitat quality, connectivity between the source and all other habitats relevant for subsequent life stages is mandatory. A high degree of connectivity is particularly crucial when species have to change habitats for spawning or during larval drift. Thereby, it is crucial to match the timing of, e.g., spawning season [48,49] or emergence, with the accessibility of those life stage specific habitats. This is particularly important if species during their ontogeny change food sources, which happens usually simultaneously with a shift in habitat requirements. Such a situation is typical for C. nasus [50,51], which obviously moved seasonally between Inn, fluvial habitats in the fish passes and the ponds in our study. Other species may follow their food sources into the habitats, such as the apex predator H. hucho [52], or use the fish pass for spawning (T. thymallus), as previously proposed by Nagel et al. [53].

Ponds contributed to fish diversity by providing habitat for additional species, which prefer stagnant water conditions, such as Tinca tinca L., S. erythrophthalmus, the strictly protected R. amarus [43,44], or the invasive P. parva [54], and M. bipartitus [55]. Whilst the presence of additional species, such as $R$. amarus, can be accomplishable, the occurrence of $M$. bipartitus is not always desired since it is known to be an invasive species potentially replacing the indigenous Misgurnus fossilis L. [55,56]. In addition to the contribution to the overall diversity of the FP that was highest during the summer months, ponds provided habitat for species of high conservation value which has previously received little attention. This peak in diversity during summer can likely be attributed to higher structural density caused by macrophytes and bank vegetation, as well as more available habitat area, due 
to higher water levels, particularly in the ponds of fish pass Gars, where, during summer, the e-flow regime is applied. Besides the small life stages of the rheophilic $C$. nasus, the stagnophilic R. amarus [45] had peak densities in these ponds during summer. Usually, in braided high-energy rivers, like the River Inn, its distribution range is restricted to backwaters and small floodplain ponds [57], which all underlie strong river dynamic processes of habitat change [58-60]. In the historically (early 1960s) highly altered River Inn, the remaining backwaters have meanwhile undergone a successional development peaking often in a terminal phase. Some of those backwaters shifted towards terrestrial habitats, and others are being used for recreational purposes, such as angling or bathing lakes. Due to the restricted river dynamic processes, which consistently would have created new backwaters and floodplain ponds, such habitats are today widely lacking. Therefore, the construction of such structures can be important. However, it has to be noted that also artificially created floodplain ponds undergo rapid successional changes, which need to be mitigated in order to preserve high species richness. Particularly macrophyte coverage increased rapidly in the rather shallow ponds since their construction, restricting habitat area for at least some species. This creates the need for a partial maintenance by clear-cutting when macrophyte coverage exceeds $75 \%$ of pond area on a regular basis.

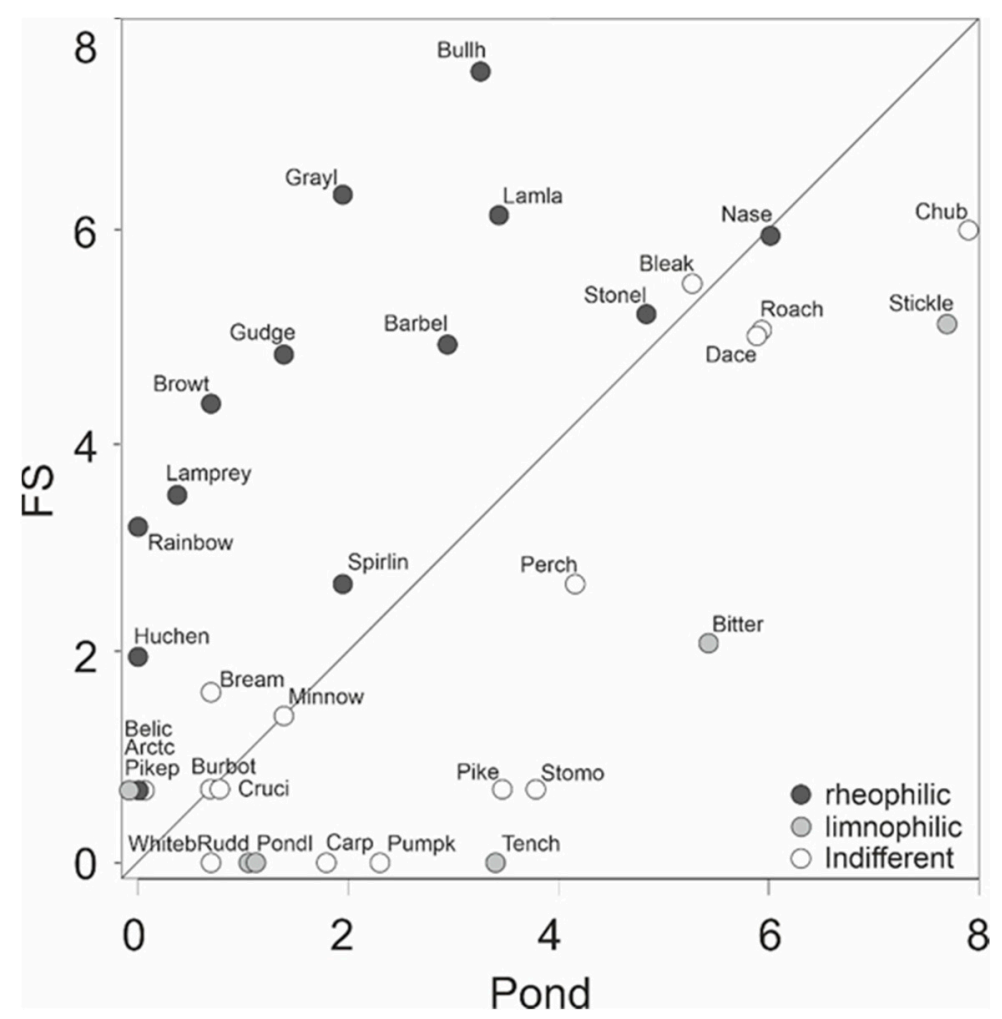

Figure 7. Scatterplot of pooled fish abundance data (based on $\log (X+1)$ transformed abundance data), distinguishing their primary occurrence in floodplain ponds and fluvial habitats (FS) of the fish passes Gars and Stammham. Shaded symbols indicate the different current preferences of species according to Reference [45]. For abbreviations of species, refer to Table 6.

The rich species inventory in the system must not obscure the fact that fish community composition in the highly degraded River Inn of today comprises a reduced set of riverine specialists that typically dominated the fish community in the past, originally comprising historically widespread and now undetected species, such as Zingel streber Siebold and Zingel zingel L. [30]. This underscores the finding that as good as the habitat quality of a fish pass might be, the species inventory largely depends on the source and if the source habitat is severely altered, an ecological integer fish community composition in restored areas or fish passes might be limited [27]. To date, it is not fully clear if the smaller size and 
discharge of a fish pass compared to the main river can still provide a suitable compensatory habitat, since it has not yet been proven if all riverine species can benefit from fish passes as permanent habitat or at least habitat for certain life stages. For example, B. barbus, Z. zingel, and Z. streber are known to prefer the strong flowing main current of rivers in their adult stages and only use riverbanks during early ontogeny [61,62]. In our study, B. barbus was present in the fish passes but only in small size-classes. Z. zingel and Z. streber could not be detected at all due to the earlier mentioned reasons for habitat degradation in the main river. If these rare species will respond positively to the installation of nature-like fish passes, they can only be determined on a long-term basis because it needs some time for them to reach a critical population size that can be detected reliably by fish population assessments (e.g., $[18,63])$.

\section{Conclusions}

This study underlines the important habitat role of fish passes, which can largely contribute to overall fish diversity in rivers, comprising typically rheophilic species in fluvial habitats and stagnophilic species in stagnant waters, such as the created floodplain ponds. Both, rheophilc and stagnophilic, species of high conservation value, such as, e.g., T. thymallus, C. nasus, B. barbus, H. hucho, and R. amarus, are rare in the main river. Some of those species, e.g., T. thymallus, even occurred in large numbers across all size classes in the fish passes, suggesting that fish passes can host a complete population of this species. Other species, which continue declining in the main river, were present in high numbers of small life stages in fluvial habitats, as well as ponds, indicating potential functionality of both habitat types as juvenile habitats. Provided regular maintenance to keep the small floodplain ponds open, high species diversity can be achieved in combination with fish passes.

Author Contributions: Conceptualization, J.P., C.N., and J.G.; methodology, J.P, C.N.; software, J.P., C.N.; validation, J.G.; formal analysis, J.P., C.N.; investigation, J.P., C.N.; resources, J.G.; data curation, J.P., C.N.; writing — original draft preparation, J.P.; writing—review and editing, C.N., J.G.; visualization, J.P.; supervision, J.G.; project administration, J.P., J.G.; funding acquisition, J.G. All authors have read and agreed to the published version of the manuscript.

Funding: This research was funded by Verbund Innkraftwerke GmbH, grant number 5810388.

Institutional Review Board Statement: This study was conducted under permit no. 31-7562 (LRA FS to the Aquatic Systems Biology Unit of TUM, valid through 15/02/2022) following standard sampling procedures to catch and handle fish in accordance with national and European guidelines [33,34] as well as the guidelines of the Declaration of Helsinki.

Informed Consent Statement: Not applicable.

Data Availability Statement: Data are available from the corresponding author upon reasonable request.

Acknowledgments: We are grateful for the financial support of this study by Verbund Innkraftwerke $\mathrm{GmbH}$, particularly to G. Loy who initiated and supported this project. We would also like to thank the angling clubs Isaria e.V. and Fischereiverein Burghausen e.V., as well as B. Gum and L. Egg (Fischereifachberatung Oberbayern) for their support of the study.

Conflicts of Interest: The authors declare no conflict of interest.

\section{References}

1. Dynesius, M.; Nilsson, C. Fragmentation and Flow Regulation of River Systems in The northern Third of the World. Science 1994, 266, 753-762. [CrossRef]

2. Bierschenk, A.M.; Mueller, M.; Pander, J.; Geist, J. Impact of Catchment Land Use on Fish Community Composition in the Headwater Areas of Elbe, Danube and Main. Sci. Total. Environ. 2019, 652, 66-74. [CrossRef] [PubMed]

3. Mueller, M.; Bierschenk, A.M.; Bierschenk, B.M.; Pander, J.; Geist, J. Effects of Multiple Stressors on the Distribution of Fish Communities in 203 Headwater Streams of Rhine, Elbe and Danube. Sci. Total. Environ. 2020, 703, 134523. [CrossRef] [PubMed] 
4. Larinier, M.; Marmulla, G. Fish Passes: Types, Principles and Geographical Distribution-an Overview. In Proceedings of the Second International Symposium on the Management of Large Rivers for Fisheries; Food and Agriculture Organization: Rome, Italy, 2004; Volume 2, pp. 183-206. Available online: http:/ /www.fao.org/3/ad526e/ad526e0g.htm (accessed on 6 April 2021).

5. Silva, A.T.; Lucas, M.C.; Castro-Santos, T.; Katopodis, C.; Baumgartner, L.J.; Thiem, J.D.; Aarestrup, K.; Pompeu, P.S.; O’Brien, G.C.; Braun, D.C.; et al. The Future of Fish Passage Science, Engineering, and Practice. Fish Fish. 2018, 19, 340-362. [CrossRef]

6. Venus, T.E.; Smialek, N.; Pander, J.; Harby, A.; Geist, J. Evaluating Cost Trade-Offs between Hydropower and Fish Passage Mitigation. Sustainability 2020, 12, 8520. [CrossRef]

7. Cubanová, L. Methodology for Fish Passes Design. “Ovidius” University Annals Constantza. Ser. Civil Eng. 2013, 15, 233-240.

8. Weyand, M.; Kühlmann, M. River Continuity Under Difficult Boundary Conditions-Useful Steps and Practical Hints for Designing a Fish Way. Water Pract. Technol. 2016, 11, 228-233. [CrossRef]

9. Calles, E.O.; Greenberg, L.A. The Use of Two Nature-Like Fishways by Some Fish Species in the Swedish River Emån. Ecol. Fish 2007, 16, 183-190. [CrossRef]

10. Baki, A.B.M.; Zhu, D.Z.; Rajaratnam, N. Flow Simulation in a Rock-Ramp Fish pass. J. Hydraul. Eng. 2016, $142,04016031$. [CrossRef]

11. Meulenbroek, P.; Drexler, S.; Nagel, C.; Geistler, M.; Waidbacher, H. The Importance of a Constructed Near-Nature-Like Danube Fish by-Pass as a Lifecycle Fish Habitat for Spawning, Nurseries, Growing and Feeding: A Long-Term View with Remarks on Management. Mar. Freshw. Res. 2018, 69, 1857-1869. [CrossRef]

12. Katopodis, C.; Kells, J.A.; Acharya, M. Nature-Like and Conventional Fishways: Alternative Concepts? Can. Water Resour. J. 2001, 26, 211-232. [CrossRef]

13. Bretón, F.; Baki, A.B.M.; Link, O.; Zhu, D.Z.; Rajaratnam, N. Flow in Nature-Like Fishway and its Relation to Fish Behaviour. Can. J. Civil. Eng. 2013, 40,567-573. [CrossRef]

14. EU Commission. Towards Sustainable Water Management in the European Union. First Stage in the Implementation of the Water Framework Directive 2000/60/EC; Commission Staff Working Document, Accompanying Document to the Communication Forum from the Commission to the European Parliament and the Council COM 2007; EU Commission: Brussels, Belgium, $2007 ;$ p. 128.

15. Kondolf, M.G.; Boulton, A.J.; O’Daniel, S.; Geoffrey, C.; Poole, G.C.; Rahel, F.J.; Stanley, E.H.; Wohl, E.; Bång, A.; Carlstrom, J.; et al. Process-Based Ecological River Restoration: Visualizing Three-Dimensional Connectivity and Dynamic Vectors to Recover Lost Linkages. Ecol. Soc. 2006, 11, 1-17. Available online: https://www.jstor.org/stable/26266026 (accessed on 6 April 2021). [CrossRef]

16. Beechie, T.J.; Sear, D.A.; Olden, J.D.; Pess, G.R.; Buffington, J.M.; Moir, H.; Roni, P.; Pollock, M.M. Process-Based Principles for Restoring River Ecosystems. BioScience 2010, 60, 209-222. [CrossRef]

17. Palmer, M.; Ruhi, A. Linkages between Flow Regime, Biota, and Ecosystem Processes: Implications for River Restoration. Science 2019, 365, 1-13. [CrossRef] [PubMed]

18. Pander, J.; Geist, J. Ecological Indicators for Measuring Stream Restoration Success. Ecol. Indic. 2013, 30, 106-118. [CrossRef]

19. Pander, J.; Geist, J. The Contribution of Different Restored Habitats to Fish Diversity and Population Development in a Highly Modified River: A Case Study from the River Günz. Water 2018, 10, 1202. [CrossRef]

20. Forseth, T.; Harby, A. Handbook for Environmental Design in Regulated Salmon Rivers; NINA Special Report; Norsk Institutt for Naturforskning: Trondheim, Norway, 2014.

21. Noonan, M.J.; Grant, J.W.; Jackson, C.D. A Quantitative Assessment of Fish Passage Efficiency. Fish. Fish 2012, 13, 450-464. [CrossRef]

22. Pander, J.; Mueller, M.; Geist, J. Ecological Functions of Fish Bypass Channels in Streams: Migration Corridor and Habitat for Rheophilic Species. River Res. Appl. 2013, 29, 441-450. [CrossRef]

23. DWA-M 509. Fischaufstiegsanlagen und Fischpassierbare Bauwerke-Gestaltung, Bemessung, Qualitätssicherung; Deutsche Vereinigung für Wasserwirtschaft, Abwasser und Abfall e.V.: Hennef, Germany, 2014; p. 334. ISBN 978-3-942964-91-3.

24. Wolter, C.; Schomaker, C. Fish Passes Design Discharge Requirements for Successful Operation. River Res. Appl. 2019, 35, 1697-1701. [CrossRef]

25. Nannini, M.A.; Goodrich, J.; Dettmers, J.M.; Soluk, D.A.; Wahl, D.H. Larval and Early Juvenile Fish Dynamics in Main Channel and Backwater Lake Habitats of the Illinois River Ecosystem. Ecol. Freshw. Fish 2012, 21, 499-509. [CrossRef]

26. Nicolas, Y.; Pont, D. Hydrosedimentary Classification of Natural and Engineered Backwaters of a Large River, the Lower Rhône: Possible Applications for the Maintenance of High Fish Biodiversity. Regul. Rivers: Res. Manag. An. Int. J. Devoted River Res. Manag. 1997, 13, 417-431. [CrossRef]

27. Pander, J.; Mueller, M.; Geist, J. Succession of Fish Diversity After Reconnecting a Large Floodplain to the Upper Danube River. Ecol. Eng. 2015, 75, 41-50. [CrossRef]

28. Garrett-Walker, J.; Collier, K.J.; Daniel, A.; Hicks, B.J.; Klee, D. Design Features of Constructed Floodplain Ponds Influence Waterbird and Fish Communities in Northern New Zealand. Freshw. Biol. 2020, 65, 2066-2080. [CrossRef]

29. Pander, J.; Mueller, M.; Knott, J.; Egg, L.; Geist, J. Is it Worth the Money? The Functionality of Engineered Shallow Stream Banks as Habitat for Juvenile Fishes in Heavily Modified Water Bodies. River Res. Appl. 2017, 33, 63-72. [CrossRef]

30. Schmall, B.; Ratschan, C. Die historische und aktuelle Fischfauna der Salzach-ein Vergleich mit dem Inn. Beitr. Naturk. Oberösterreichs 2011, 21, 55-191. 
31. Arthington, A.H.; Bhaduri, A.; Bunn, S.E.; Jackson, S.E.; Tharme, R.E.; Tickner, D.; Young, B.; Acreman, M.; Baker, N.; Capon, S.; et al. The Brisbane Declaration and Global Action Agenda on Environmental Flows. Front. Environ. Sci. 2018, 6, 15. [CrossRef]

32. Pander, J.; Geist, J. Seasonal and Spatial Bank Habitat Use by Fish in Highly Altered Rivers-a Comparison of Four Different Restoration Measures. Ecol. Freshw. Fish 2010, 19, 127-138. [CrossRef]

33. CEN. Water Quality-Sampling Fish with Electricity; CEN prEN: Brussels, Belgium, 2003; p. 16.

34. Dußling, U. Handbuch zu fiBS; Schriftenreihe des Verbandes Deutscher Fischereiverwaltungsbeamter und Fischereiwissenschaftler e.V.: Rostock, Germany, 2009; p. 72.

35. Mueller, M.; Pander, J.; Geist, J. Comprehensive Analysis of $>30$ Years of Data on Stream Fish Population Trends and Conservation Status in Bavaria, Germany. Biol. Conserv. 2018, 226, 311-320. [CrossRef]

36. Clarke, K.R.; Gorley, R.N.; Somerfield, P.J.; Warwick, R.M. Change in Marine Communities: An Approach to Statistical Analysis and Interpretation, 3rd ed.; PRIMER-E: Plymouth, UK, 2014; p. 144.

37. Bray, J.R.; Curtis, J.T. An Ordination of the Upland Forest Communities of Southern Wisconsin. Ecol. Monogr. 1957, 27, 325-349. [CrossRef]

38. Clarke, K.R. Non-Parametric Multivariate Analyses of Changes in Community Structure. Aust. J. Ecol. 1993, 18, 117-143. [CrossRef]

39. Clarke, K.R.; Somerfield, P.J.; Chapman, M.G. On Resemblance Measures for Ecological Studies, Including Taxonomic Dissimilarities and a Zero-Adjusted Bray-Curtis Coefficient for Denuded Assemblages. J. Exp. Mar. Biol. Ecol. 2006, 330, 55-80. [CrossRef]

40. Hartig, F. DHARMa: Residual Diagnostics for Hierarchical (Multi-Level/Mixed) Regression Models. R Package Version 0.3.3.0. 2020. Available online: http:/ / florianhartig.github.io/DHARMa/ (accessed on 18 February 2021).

41. R Core Team R. A Language and Environment for Statistical Computing; R Foundation for Statistical Computing: Vienna, Austria, 2017; Available online: https:/ / www.R-project.org/ (accessed on 18 February 2021).

42. Fox, J.; Weisberg, S. An R Companion to Applied Regression, 2nd ed.; Sage Publications: Thousand Oaks, CA, USA, 2018 ; p. 571.

43. Bohl, E.; Kleisinger, H.; Leuner, E. Rote Liste gefährdeter Fische (Pisces) und Rundmäuler (Cyclostomata) Bayern. Bayerisches Landesamt für Umwelt, BayLfU/166/2003. 2003. Available online: https:/ /www.sf-kemmern.de/pdf_Dateien/rote_liste.pdf (accessed on 18 February 2021).

44. Council of the European Communities. Council Directive 92/43/EEC of 21 May 1992 on the Conservation of Natural Habitats and of Wild Fauna and Flora. Off. J. Eur. Union 1992, 206, 7-50.

45. Zauner, G.; Eberstaller, J. Klassifizierungsschema der Österreichischen Flußfischfauna in Bezug auf Deren Lebensraumansprüche. Osterr. Fischerei 1999, 52, 198-205.

46. Bohlin, T.; Hamrin, S.; Heggberget, T.G.; Rasmussen, G.; Saltveit, S.J. Electrofishing-Theory and Practice with Special Emphasis on Salmonids. Hydrobiologia 1989, 173, 9-43. [CrossRef]

47. Mollenhauer, R.; Logue, D.; Brewer, S.K. Quantifying Seining Detection Probability for Fishes of Great Plains Sand-Bed Rivers. Trans. Am. Fish. Soc. 2018, 147, 329-341. [CrossRef]

48. Cushing, D.H. Plankton Production and Year-Class Strength in Fish Populations: An Update of the Match/Mismatch Hypothesis. Adv. Mar. Biol. 1990, 26, 249-293.

49. Sternecker, K.; Denic, M.; Geist, J. Timing Matters: Species-Specific Interactions between Spawning Time, Substrate Quality, and Recruitment Success in Three Salmonid Species. Ecol. Evol. 2014, 4, 2749-2758. [CrossRef]

50. Reinartz, R. Untersuchungen zur Gefährdungssituation der Fischart Nase (Chondrostoma nasus L.) in Bayerischen Gewässern. Ph.D. Thesis, Technische Universität München, München, Germany, 1997.

51. Reckendorfer, W.; Keckeis, H.; Tiitu, V.; Winkler, G.; Zornig, H. Diet shifts in 0+ nase, Chondrostoma nasus: Size-Specific Differences and the Effect of Food. Arch. Hydrobiol. Suppl. 2001, 13512, 425-440. [CrossRef]

52. Holcčík, J. Threatened Fishes of the World: Hucho Hucho (Linnaeus, 1758) (Salmonidae). Environ. Biol. Fishes 1995, 43, 105-106. [CrossRef]

53. Nagel, C.; Mueller, M.; Pander, J.; Stoeckle, B.C.; Kuehn, R.; Geist, J. Going with the Flow: Spatio-Temporal Drift Patterns of Larval Fish in a Large Alpine River. Freshw. Biol. 2021. Under Review.

54. Britton, J.R.; Davies, G.D.; Harrod, C. Trophic Interactions and Consequent Impacts of the Invasive Fish Pseudorasbora Parva in a Native Aquatic Foodweb: A Field Investigation in the UK. Biol. Invasions 2010, 12, 1533-1542. [CrossRef]

55. Zangl, L.; Jung, M.; Gessl, W.; Koblmueller, S.; Ratschan, C. Oriental or Not: First Record of an Alien Weatherfish (Misgurnus) Species in Austria Verified by Molecular Data. BioInvasions Rec. 2020, 9, 375-383. [CrossRef]

56. Stoeckle, B.C.; Belle, C.C.; Geist, J.; Oehm, J.; Effenberger, M.; Heiss, M.; Seifert, K.; Kuehn, R. Molecular Confirmation of the Large-Scale Loach Paramisgurnus Dabryanus Dabry de Thiersant, 1872 (Cypriniformes, Cobitidae) in Europe. BioInvasions Rec. 2019, 8, 419-426. [CrossRef]

57. Schomaker, C.; Wolter, C. The Contribution of Long-Term Isolated Water Bodies to Floodplain Fish Diversity. Freshw. Biol. 2011, 56, 1469-1480. [CrossRef]

58. Tockner, K.; Ward, J.V.; Arscott, D.B.; Edwards, P.J.; Kollmann, J.; Gurnell, A.M.; Petts, G.E.; Maiolini, B. The Tagliamento River: A Model Ecosystem of European Importance. Aquat. Sci. 2003, 65, 239-253. [CrossRef]

59. Van Der Nat, D.; Tockner, K.; Edwards, P.J.; Ward, J.V.; Gurnell, A.M. Habitat Change in Braided Flood Plains (Tagliamento, NE-Italy). Freshw. Biol. 2003, 48, 1799-1812. [CrossRef] 
60. Schiemer, F.; Drescher, A.; Hauer, C.; Schwarz, U. The Vjosa River Corridor: A Riverine Ecosystem of European Significance. Acta Zoo Bot. Austria 2018, 155, 1-40.

61. Telcean, I.C.; Cupşa, D. Fishfauna from the Lowland Mureş River (Romania) and the Floodplain Natural Park Area (Western Romania). An. Univ. Oradea Fasc. Biol. 2009, 16, 131-135.

62. Britton, J.R.; Pegg, J. Ecology of European barbel Barbus Barbus: Implications for River, Fishery, and Conservation Management. Rev. Fish. Sci. 2011, 19, 321-330. [CrossRef]

63. Kottelat, M.; Freyhof, J. Handbook of European Freshwater Fishes; Publications Kottelat: Cornol, Switzerland, $2007 ;$ p. 646. 